\title{
Experimental Characterization and Mathematical Modeling of P2X7 Receptor Channel Gating
}

\author{
Zonghe Yan, ${ }^{1 *}$ Anmar Khadra, ${ }^{2 \star}$ Shuo Li, ${ }^{1}$ Melanija Tomić, ${ }^{1}$ Arthur Sherman, ${ }^{2}$ and Stanko S. Stojilkovic ${ }^{1}$ \\ ${ }^{1}$ Section on Cellular Signaling, Program in Developmental Neuroscience, National Institute of Child Health and Human Development, and ${ }^{2}$ Laboratory of \\ Biological Modeling, National Institute of Diabetes and Digestive and Kidney Diseases, National Institutes of Health, Bethesda, Maryland $20892-4510$
}

The P2X7 receptor is a trimeric channel with three binding sites for ATP, but how the occupancy of these sites affects gating is still not understood. Here we show that naive receptors activated and deactivated monophasically at low and biphasically at higher agonist concentrations. Both phases of response were abolished by application of Az10606120, a P2X7R-specific antagonist. The slow secondary growth of current in the biphasic response coincided temporally with pore dilation. Repetitive stimulation with the same agonist concentration caused sensitization of receptors, which manifested as a progressive increase in the current amplitude, accompanied by a slower deactivation rate. Once a steady level of the secondary current was reached, responses at high agonist concentrations were no longer biphasic but monophasic. Sensitization of receptors was independent of $\mathrm{Na}^{+}$and $\mathrm{Ca}^{2+}$ influx and $\sim 30$ min washout was needed to reestablish the initial gating properties. T15E- and T15K-P2X7 mutants showed increased sensitivity for agonists, responded with monophasic currents at all agonist concentrations, activated immediately with dilated pores, and deactivated slowly. The complex pattern of gating exhibited by wild-type channels can be accounted for by a Markov state model that includes negative cooperativity of agonist binding to unsensitized receptors caused by the occupancy of one or two binding sites, opening of the channel pore to a low conductance state when two sites are bound, and sensitization with pore dilation to a high conductance state when three sites are occupied.

\section{Introduction}

The P2X7 receptor (P2X7R) is a member of the family of ATPgated nonselective cation channels expressed in a large variety of cells, including microglia and macrophages (Di Virgilio et al., 2009b; Skaper et al., 2010). Although numerous studies have been performed with recombinant P2X7R since its cloning (Surprenant et al., 1996), its gating properties are not well understood. Activation of P2X7R was reported to be monophasic (Pelegrin and Surprenant, 2006) and biphasic (monoexponential plus linear phase or even more kinetically complex secondary phase) (Chessell et al., 2001; Klapperstück et al., 2001; Smart et al., 2003; Yan et al., 2008). Current deactivation was suggested to follow a monoexponential time course with time constants $<1 \mathrm{~s}$ (Klapperstück et al., 2000) but also biexponential and nonexponential time courses with slow kinetics (Petrou et al., 1997; Rassendren et al., 1997; Yan et al., 2006). In addition to opening of an intrinsic cation-conducting pore, permitting the permeation of monovalent and divalent cations during a brief agonist application and causing plasma membrane depolarization, pro-

\footnotetext{
Received May 10, 2010; revised Aug. 23, 2010; accepted Aug. 30, 2010.

We were supported by the Intramural Research Program of the National Institutes of Health, National Institute of Child Health and Human Development (Z.Y., S.L., S.S.S.), and National Institute of Diabetes and Digestive and Kidney Diseases (A.K., A.S.). Z.Y. performed the bulk of the experiments and A.K. did the modeling

*Z.Y. and A.K. contributed equally to this work.

Correspondence should be addressed to Dr. Stanko S. Stojilkovic; National Institute of Child Health and Human Development, Building 49, Room 6A-36, 49 Convent Drive, Bethesda, MD 20892-4510. E-mail: stankos@helix. nih.gov or stojilks@mail.nih.gov.

DOI:10.1523/JNEUROSCI.2390-10.2010

Copyright $\odot 2010$ the authors $\quad 0270-6474 / 10 / 3014213-12 \$ 15.00 / 0$
}

longed or repetitive agonist application promotes the formation of large pores or pathways allowing the bidirectional passage of organic cations of up to $900 \mathrm{Da}$ and the leakage of metabolites (North, 2002).

At least two conflicting hypotheses have been postulated to reconcile these findings: (1) The pore-dilation hypothesis suggests that there is a progressive dilation of the cation-conducting pore from initially $7 \AA$ up to $40 \AA$, as observed in whole-cell recording in cells bathed in medium containing large organic cations instead of sodium (Virginio et al., 1999). (2) The two-pore hypothesis implies the activation of an endogenous P2X7R pore permeable for inorganic cations, accompanied by sustained activation of a distinct channel permeable to larger organic cations and fluorescent dyes. The lack of a molecular correlate to pore dilation at the single P2X7R channel level (Riedel et al., 2007), the presence of two distinct ATP binding sites on P2X7R (Klapperstück et al., 2001), and the finding that the P2X7R-dependent fluorescent dye uptake correlates with recruitment of pannexin-1 hemichannels (Pelegrin and Surprenant, 2006; Locovei et al., 2007) or a modulation of the multidrug transporter P-glycoprotein (Elliott et al., 2005) support the second hypothesis. Both hypotheses provide a rationale for the observation by many laboratories that activation of these receptors causes cell death (Surprenant and North, 2009), but neither explains the finding that activation of these receptors causes cell growth and differentiation (Di Virgilio et al., 2009a; Monif et al., 2009).

Here we address how the occupation of three P2X7R binding sites affects gating. Using an ultra-fast solution-switching system with a time resolution of $\sim 1 \mathrm{~ms}$ to minimize the side effects of 
agonist application, we show that rat P2X7R can activate and deactivate either monophasically, with almost constant amplitude and deactivation kinetics, or biphasically, with increased current amplitude and slowed deactivation. Repeated exposure results in monophasic kinetics, but with large current amplitude and slow deactivation locked in. We also provide a mathematical model that can account for this complex pattern of gating.

\section{Materials and Methods}

Cell culture, transfection, and constructs. HEK293 and GT1 cells were used for the expression of various P2X7R constructs, as described previously (Yan et al., 2006). HEK293 cells (obtained from American Type Culture Collection) were routinely maintained in DMEM containing 10\% (v/v) fetal bovine serum (Invitrogen) and 1\% (v/v) penicillin-streptomycin liquid (Invitrogen) in a tissue culture incubator. GT1 cells (provided by Dr. Richard I. Weiner, University of California, San Francisco) were cultured in DMEM/Ham's F-12 medium (1:1), containing 10\% (v/v) fetal bovine serum and $100 \mu \mathrm{g} / \mathrm{ml}$ gentamicin (Invitrogen). For electrophysiological measurements, HEK293 cells were grown on $35 \mathrm{~mm}$ dishes at a density of $0.5 \times 10^{6}$ cells per dish, whereas for imaging studies GT1 cells were grown on $25 \mathrm{~mm}$ coverslips placed in $35 \mathrm{~mm}$ dishes at a density of $0.1 \times 10^{6}$ cells per dish. Transfection was conducted $24 \mathrm{~h}$ after plating the cells using $2 \mu \mathrm{g}$ of DNA and $5 \mu \mathrm{l}$ of Lipofectamine 2000 reagent (Invitrogen) in $2 \mathrm{ml}$ of serum-free Opti-MEM. After $4.5 \mathrm{~h}$ of incubation, the transfection mixture was replaced with normal culture medium and cells were cultured for an additional 24-48 h. Transfected cells were mechanically dispersed and recultured on $35 \mathrm{~mm}$ dishes of Corning 3294 CellBIND Surface for $2-8 \mathrm{~h}$ before electrophysiological recording. The rat P2X7-pIRES2-EGFP construct (He et al., 2003) was used for the generation of other constructs. Plasmids containing specific amino acid point mutations of $\mathrm{P} 2 \mathrm{X} 7 \mathrm{cDNA}$ were generated using Quik-Change II XL site-directed mutagenesis kit (Stratagene), as previously described (Yan et al., 2008). The mutagenic oligonucleotide primers were synthesized and PAGE purified by Integrated DNA Technology. Production of the correct constructs was verified by dye terminator-cycle sequencing (performed by Macrogen USA). Large-scale plasmid DNAs were prepared using a QIAfilter Plasmid Maxi kit (QIAGEN).

Current measurements. Whole-cell patch-clamp recording was performed on single cells at room temperature using an Axopatch 200B amplifier (Molecular Devices) as described previously (Yan et al., 2005). Patch electrodes, fabricated from borosilicate glass (type 1B150F-3; World Precision Instruments) using a Flaming Brown horizontal puller (P-97; Sutter Instruments), were heat-polished to a final tip resistance of 3.5-5.0 M $\Omega$. All current records were captured, and stored using the pClamp 8.0 software packages in conjunction with the Digidata 1322A A/D converter (Molecular Devices). Experiments were performed on single cells with an average capacitance of $10 \mathrm{pF}$. For current recording, membrane potential was held at $-60 \mathrm{mV}$. Current voltage relations were used to estimate changes in reversal potential during agonist application and were obtained by voltage ramps from $-80 \mathrm{mV}$ to $+80 \mathrm{mV}$ twice per second during $40 \mathrm{~s}$. Patch electrodes were filled with solution containing $145 \mathrm{~mm} \mathrm{NaCl}, 10 \mathrm{~mm}$ EGTA, $20 \mathrm{~mm} \mathrm{~K}^{+}$(originating from the $0.5 \mathrm{M}$ EGTA/1 м KOH stock solution), and 10 mм HEPES; the $\mathrm{pH}$ was adjusted with $10 \mathrm{M} \mathrm{NaOH}$ to 7.35. The osmolarity of this solution was $293 \mathrm{mOsM}$. The regular Krebs-Ringer-like (KR) bath buffer contained $147 \mathrm{mM} \mathrm{NaCl}$, $3 \mathrm{~mm} \mathrm{KCl}, 1 \mathrm{~mm} \mathrm{MgCl}, 2$ mm CaCl 2,10 mm glucose, and 10 mm HEPES; the $\mathrm{pH}$ was adjusted to 7.35 with $10 \mathrm{M} \mathrm{NaOH}$. All the buffers with $10 \mathrm{~mm}$ HEPES contained additional $2.8 \mathrm{mM} \mathrm{Na}^{+}$, which originated from the $1 \mathrm{M}$ HEPES stock solution $\mathrm{pH}$-adjusted with $\mathrm{NaOH}$ (Mediatech). The osmolarity of these solutions was 289-295 mOsM. ATP, Bz-ATP and AZ10606129 (Tocris Bioscience) solution was prepared daily in bath buffer with $\mathrm{pH}$ properly readjusted, applied using either an Ultrafast Solution-Switching System (LSS-3200; EXFO Burleigh Products Group Inc.) that was simultaneously program-controlled by pClamp 8.0 software through PZ-150M Amplifier (Yan et al., 2006) or an RSC-200 Rapid Solution Changer (Biologic, France) (Yan et al., 2008). Cells with EGFP fluorescence were identified before immersing the electrode in bath solution for gigaohm seal.
Calculations. Nonlinear curve fitting of currents was performed with Clampfit 10.0 (Molecular Devices) predefined functions of exponential standard $\left(f(t)=A_{1} \exp \left(-t / \tau_{1}\right)+A_{2} \exp \left(-t / \tau_{2}\right)+C\right)$ for deactivation fitting, and exponential power $(f(t)=A(1-\exp (-t / \tau))+C)$ for the rapid first phase and Hill 4 parameter logistic function $\left(f(t)=I_{\min }+\right.$ $\left(I_{\max }-I_{\min }\right) /\left(1+\left(\tau_{2} / t\right)^{\mathrm{h}}\right)$ for the second slow-phase activation fitting; $\tau_{2}$ indicates the half-time of activation. Whenever appropriate, the data were presented as mean \pm SEM values. Significant differences, with $p<0.01$, were determined by Mann-Whitney test using GraphPad InStat 3.05.

Calcium measurements. Transfected GT1 cells plated on $25 \mathrm{~mm}$ coverslips were bathed in KR medium containing $2.5 \mu \mathrm{M}$ Fura-FF AM (Invitrogen) for $1 \mathrm{~h}$ at room temperature. After washing the coverslips with the dye-free Krebs-Ringer medium, they were mounted on the stage of an Axiovert 135 microscope (Carl Zeiss) attached to an Attofluor Digital Fluorescence Microscopy System (Atto Instruments). Cells were examined under a 40x oil-immersion objective during exposure to alternating 340 and $380 \mathrm{~nm}$ excitation beams, and the intensity of light emission at $520 \mathrm{~nm}\left(F_{340}\right.$ and $\left.F_{380}\right)$ was followed in several single cells simultaneously at the rate of one point per second.

Mathematical model. We use a Markov state model consisting of 8 states (see Fig. 7), each of which corresponds to the fraction of P2XRs that are bound to at most three ligand molecules (equations are fit to data with BzATP) and may additionally be sensitized (see Fig. 6, bottom row). We aimed for the simplest model with the minimal number of parameters needed to reproduce the main features of the data, such as monophasic and biphasic kinetics and a persistent sensitized state. For example, motivated by considerations of symmetry (see below), we have constrained the model such that all backward rate constants along the bottom row of the diagram are equal to the rate from $C_{2}$ to $C_{1}$ and all the forward rate constants are equal to the rate from $C_{1}$ to $C_{2}$, although using different rates might have produced more perfect fits. Corresponding to this scheme is the following system of 8 linear ordinary differential equations:

$$
\begin{gathered}
\frac{d C_{1}}{d t}=k_{1} C_{2}+L_{1} C_{4}-3 k_{2} A C_{1}, \\
\frac{d C_{2}}{d t}=3 k_{2} A C_{1}+2 k_{3} Q_{1}-\left(k_{1}+2 k_{4 A}\right) C_{2}, \\
\frac{d Q_{1}}{d t}=2 k_{4} A C_{2}+3 k_{5} Q_{2}-\left(2 k_{3}+k_{6} A\right) Q_{1}, \\
\frac{d Q_{2}}{d t}=k_{6} A Q_{1}+L_{2} Q_{3}-\left(3 k_{5}+L_{3}\right) Q_{2}, \\
\frac{d Q_{3}}{d t}=k_{2} A Q_{4}+L_{3} Q_{2}-\left(3 k_{1}+L_{2}\right) Q_{3}, \\
\frac{d Q_{4}}{d t}=2 k_{2} A C_{3}+3 k_{1} Q_{3}-\left(2 k_{1}+k_{2} A\right) Q_{4}, \\
\frac{d C_{3}}{d t}=3 k_{2} A C_{4}+2 k_{1} Q_{4}-\left(k_{1}+2 k_{2} A\right) C_{3}, \\
\frac{d C_{4}}{d t}=k_{1} C_{3}-\left(L_{1}+3 k_{2} A\right) C_{4} .
\end{gathered}
$$

The whole-cell current is given by the equation,

$$
l=g_{12}\left(Q_{1}+Q_{2}\right)(V-E)+g_{34}\left(Q_{3}+Q_{4}\right)(V-E),
$$

where $g_{12}$ and $g_{34}$ are the total channel conductances, $E$ is the reversal potential, and $V$ is the holding potential ( $-60 \mathrm{mV}$ in all the simulations). The parameter values are listed in Table 1. 
Table 1. Parameter values and distributions used in modeling of P2X7R gating

\begin{tabular}{|c|c|c|}
\hline \multirow[b]{2}{*}{ Symbol } & \multicolumn{2}{|c|}{ Parameter values and distributions } \\
\hline & Values & Distribution \\
\hline$k_{1}$ & $0.3 s^{-1}$ & Normal, $\sigma=0.03$ \\
\hline$k_{2}$ & 40000 (M.s) $)^{-1}$ & Normal, $\sigma=4000$ \\
\hline$k_{3}^{2}$ & $2.4 s^{-1}$ & Normal, $\sigma=0.24$ \\
\hline$k_{4}$ & $50000(\text { M.s. })^{-1}$ & Normal, $\sigma=5000$ \\
\hline$k_{5}$ & $1.58 s^{-1}$ & Normal, $\sigma=0.158$ \\
\hline$k_{6}$ & $7000(\text { M.s })^{-1}$ & Normal, $\sigma=700$ \\
\hline$L_{1}$ & $0.0001 s^{-1}$ & \\
\hline$L_{2}$ & $0.004 s^{-1}$ & Normal, $\sigma=4 \times 10^{-4}$ \\
\hline$L_{3}$ & $0.5,0.1 s^{-1}$ & Uniform, $[0.1-3]$ \\
\hline$g_{12}\left(Q_{1}+Q_{2}\right.$ conductance $)$ & $1.5 \times 10^{-8} \mathrm{~S}$ & Normal, $\sigma=1.5 \times 10^{-9}$ \\
\hline$g_{34}\left(Q_{3}+Q_{4}\right.$ conductance $)$ & $4.5 \times 10^{-8} \mathrm{~S}$ & Normal, $\sigma=4.5 \times 10^{-9}$ \\
\hline$E$ (reversal potential) & $0 \mathrm{~V}$ & Normal, $\sigma=1$ \\
\hline
\end{tabular}

\section{Results}

\section{Concentration dependence of the growth of two current and} calcium components

Experiments on currents were performed with wild-type rat P2X7Rs expressed in HEK293 cells bathed in KR buffer containing $2 \mathrm{mM} \mathrm{Ca}^{2+}$. All experiments were performed in cells not previously stimulated with agonists and all recordings were made in one cell per dish during a single agonist application/withdrawal of variable duration. Figure 1 illustrates the patterns of current responses in cells stimulated with ATP (A) and BzATP (B) for 40 s or longer. Monophasic, slowly developing currents of small amplitude were always observed in cells stimulated with $100 \mu \mathrm{M}$ ATP and 3.2 $\mu \mathrm{M}$ BzATP. Such currents were also consistently observed in response to $320 \mu \mathrm{M}$ ATP application, and in a fraction of cells stimulated with $10 \mu \mathrm{M}$ BzATP, but the rise in currents was faster and amplitudes were higher than currents generated by $100 \mu \mathrm{M}$ ATP and $3.2 \mu \mathrm{M}$ BzATP. At higher agonist concentrations (1-10 mM ATP and 32-320 $\mu \mathrm{M}$ BzATP), we always observed biphasic currents in naive cells; the initial rise in current $\left(I_{1}\right)$ was accompanied by secondary current growth $\left(I_{2}\right)$, and the rate of $I_{2}$ growth increased with elevation in agonist concentrations (Fig. 1A, $B$ ).

The dose dependence of BzATP and ATP effects on the total $\left(I_{1}+I_{2}\right)$ current in naive cells reached at $40 \mathrm{~s}$ stimulation indicated an $\sim 55$-fold difference in the potency of the two agonists (Fig. 1C), which is in accordance with the literature (North, 2002). Consistent with the lower affinity of P2X7Rs to ATP, the washout of this agonist was accompanied by a rapid decline of current (deactivation), whereas the decline of the current after washout of the more potent BzATP was slower. Figure 1, $A$ and $B$, also shows that the ratio between $I_{1}$ and $I_{2}$ amplitudes changed with increase in agonist concentrations, as did the time needed to reach steady state. The mean \pm SEM values of $I_{1}$ and $I_{2}$ amplitudes, the latter measured after $40 \mathrm{~s} \mathrm{BzATP}$ application, also show that the $I_{1}$ current amplitude increased progressively with elevation in agonist concentration, whereas the $I_{2}$ amplitude first increased and then decreased with increase in agonist concentration (Fig. 1D).

Previous experiments with Fura-2 failed to show high intracellular calcium concentration $\left(\left[\mathrm{Ca}^{2+}\right]_{\mathrm{i}}\right)$ in cells with activated P2X7Rs (Koshimizu et al., 2000), prompting us to use the less sensitive Fura-FF dye. GT1 cells were used as an expression system, because HEK293 cells endogenously express $\mathrm{Ca}^{2+}$ mobilizing P2Y receptors (He et al., 2003). In GT1 cells, BzATP induced concentration-dependent increase in $\left[\mathrm{Ca}^{2+}\right]_{\mathrm{i}}$ (Fig. $1 E$ ). At lower agonist concentrations, a slow and monophasic rise in $\left[\mathrm{Ca}^{2+}\right]_{\mathrm{i}}$ was observed, reaching the plateau response after 2-4 min of application. At intermediate concentrations, BzATP induced biphasic $\left[\mathrm{Ca}^{2+}\right]_{\mathrm{i}}$ responses, labeled as $\mathrm{Ca}_{1}$ and $\mathrm{Ca}_{2}$, with the rates determined by agonist concentration. As described previously, sustained BzATP causes leak of Fura-FF (Yan et al., 2008), which limits interpretation of the peak amplitude of $\left[\mathrm{Ca}^{2+}\right]_{\mathrm{i}}$ in response to $20-100 \mu \mathrm{M}$ BzATP (indicated by dashed line).

In Figure 2, we analyze in detail the kinetics of current growth and decay during the initial agonist application and washout. In all concentrations, the $I_{1}$ rise was best fitted with a monoexponential function. Representative traces for such fitting are illustrated in Figure $2 \mathrm{~A}$, with the calculated time constants $\left(\tau_{1}\right)$ shown above the traces. Notice that the rate of $I_{1}$ growth increased with elevation in BzATP concentration. On the other hand, the fourparameter logistic function, commonly used for the description of concentration responses, was the best fit for the $I_{2}$ growth in all concentrations. Representative traces of such fitting for the $I_{2}$ are shown in Figure $2 B$, with the vertical dotted lines indicating the half time of activation $\left(\tau_{2}\right)$. We used the mean \pm SEM values of constants $\tau_{1}$ and $\tau_{2}$ to show dependence of the activation time courses of the P2X7R on BzATP concentrations (Fig. 2D). This analysis showed that the time for activation of both currents decreased with increase in BzATP concentrations, with a fivefold difference in the $\mathrm{EC}_{50}$ values.

The best approximation for decay of current after the washout of agonist was achieved using monoexponential or biexponential fittings, depending on agonist concentration (Fig. 2C). The decay constants were denoted $\tau_{\mathrm{f}}$ (fast) and $\tau_{\mathrm{s}}$ (slow) for biphasic responses and $\tau_{\mathrm{s}}$ for monoexponential decays. In general, when the activation kinetics was best described by monoexponential rise, the deactivation kinetics could also be approximated well by a monoexponential decay, and the decay of currents was always biexponential when the secondary current growth was present. For biphasic currents triggered by $100 \mu \mathrm{M}$ BzATP, $\tau_{\mathrm{s}}$ was $14.3 \pm$ $1.4 \mathrm{~s}$ and $\tau_{\mathrm{f}}$ was $2.9 \pm 0.6 \mathrm{~s}(n=18)$ and for $3.2 \mathrm{~mm} \mathrm{ATP} \tau_{\mathrm{s}}$ was $2.4 \pm 0.3 \mathrm{~s}$ and $\tau_{\mathrm{f}}$ was $0.5 \pm 0.1 \mathrm{~s}(n=16)$. Exponential fitting of deactivation curves also permitted estimates of $\mathrm{I}_{\mathrm{f}}$ and $\mathrm{I}_{\mathrm{s}}$ amplitudes at different agonist concentrations; the mean \pm SEM values are shown in Figure $2 E$ and the ratio between $\mathrm{EC}_{50}$ values for two currents was $\sim 1: 5$.

\section{Effects of NMDG and Az10606120 on agonist-induced current response}

Substitution of bath $\mathrm{Na}^{+}$with NMDG permits both current recordings in cells held at a fixed potential and detection of changes in reversal potential estimated by repetitive voltage ramp pulses from $-80 \mathrm{mV}$ to $+80 \mathrm{mV}$ and was used to support the hypothesis of P2X7R pore dilation (Yan et al., 2008). An example of such experiments is shown in Figure $3 A-C$. Naive cells bathed in NMDG-containing medium and held at $-60 \mathrm{mV}$ responded to $100 \mu \mathrm{M}$ BzATP application with generation of biphasic currents during the initial $40 \mathrm{~s}$ application, and the washout of agonist was accompanied by slow deactivation of receptors (A). Currentvoltage curves constructed from voltage ramp pulses delivered twice per second showed a positive shift in reversal potential from $\sim-36 \mathrm{mV}$ to $-22 \mathrm{mV}$ (B). The rate of shift in reversal potential was highly comparable to the rate of $I_{2}$ current growth ( $C$ ), suggesting that during initial agonist application, the $I_{2}$ growth reflects pore dilation rather than integration of another ionic conductance.

To determine whether the slow $I_{2}$ current component was due to a single pore or involved the opening of a second pore, we studied the effects of Az10606120, a P2X7R-specific antagonist 
A

0.1

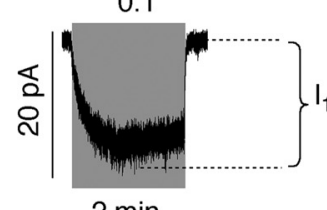

2 min
0.32

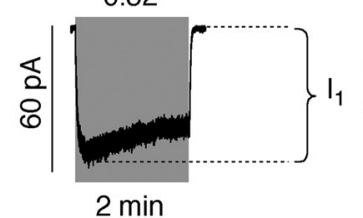

$2 \min$

B

3.2

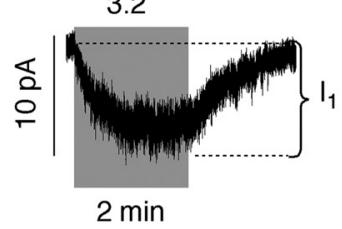

10

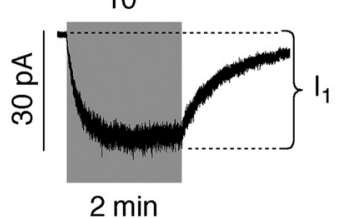

ATP (mM):

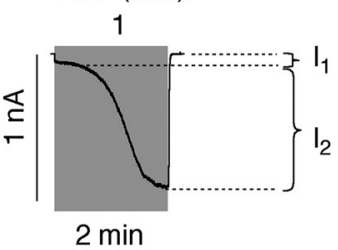

2 min

ZATP $(\mu \mathrm{M})$

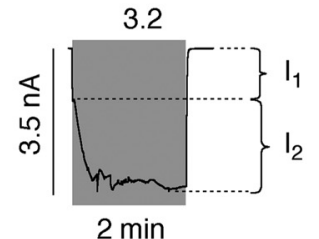

2 min

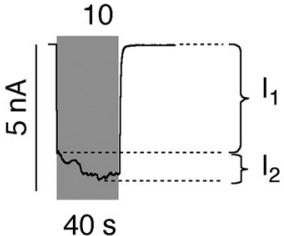

$40 \mathrm{~s}$
32

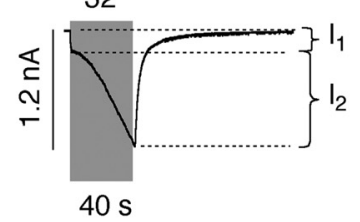

100

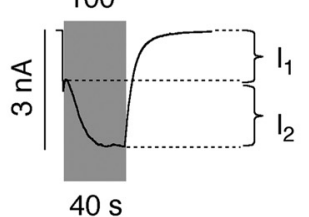

320

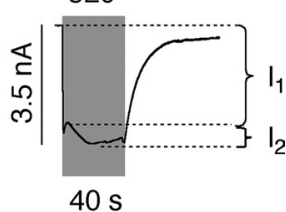

C

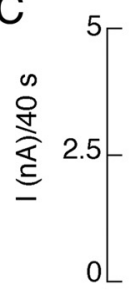

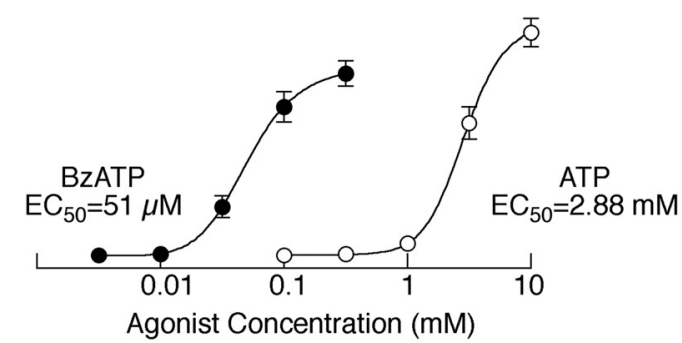

D

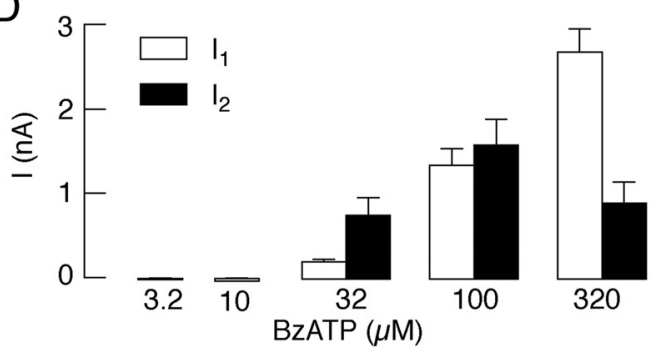

E

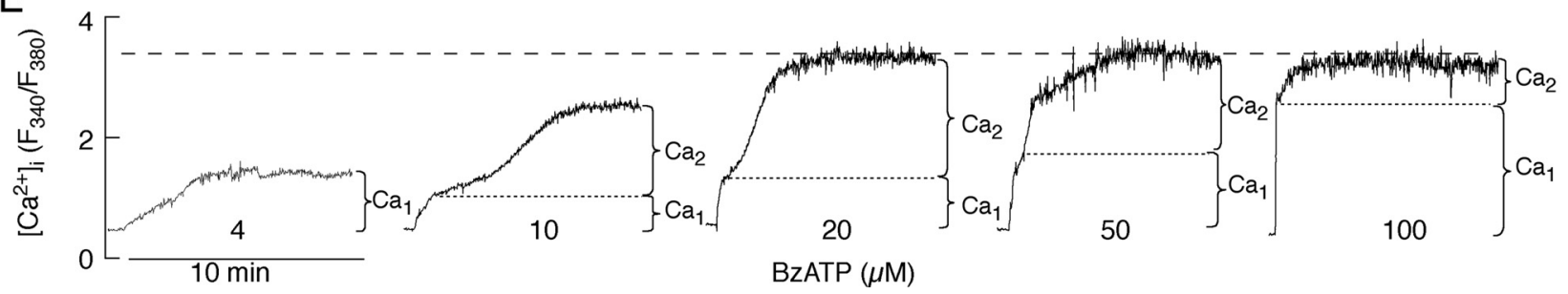

Figure 1. Monophasic and biphasic activation of naive rat P2X7R expressed in HEK293 cells. $A, B$, Concentration-dependent effects of ATP $(A)$ and BzATP $(B)$ on receptor activation. In this and the following figures, current recordings were made in cells bathed in KR buffer containing $2 \mathrm{~mm}$ calcium and with $\mathrm{Ca}^{2+}$-deficient pipette medium containing $10 \mathrm{~mm}$ EGTA, and cells were held at $-60 \mathrm{mV}$, if not otherwise specified. Traces shown are representative for at least five records per dose. Gray areas indicate duration of agonist application. $I_{1}$, the primary current; $I_{2}$, the secondary current. Notice that the current scales are variable, and that there is a transient decline of the $I_{1}$ in response to 100 and $320 \mu \mathrm{m}$ BZATP application. C, Concentration dependence of ATP (open circles) and BzATP (closed circles) on the peak amplitude of current reached during $40 \mathrm{~s}$ stimulation. $D$, Concentration dependence of the $I_{1}$ and $I_{2}$ amplitudes on BzATP, the second measured after 40 s agonist application. In C and D, data shown are means \pm SEM from at least 5 records per dose, each performed in separate cells during initial agonist application. $\boldsymbol{E}$, Concentration-dependent effect of BzATP on the rate of calcium influx and the peak amplitude of calcium signals in GT1 cells expressing recombinant P2X7R. $\mathrm{Ca}_{1}$ and $\mathrm{Ca}_{2}$, the primary and secondary response. Traces shown are representative from at least 20 records per dose.

(Michel et al., 2007), on the initiation and duration of $I_{1}$ and $I_{2}$. When $1 \mu \mathrm{M}$ Az10606120 was applied for $20 \mathrm{~s}$ before addition of $100 \mu \mathrm{M}$ BzATP, no current response was observed (Fig. 3E, fourth application, Fig. 3G, second application). However, when $100 \mathrm{~nm}$ Az10606120 and $100 \mu \mathrm{M}$ BzATP were applied together, a rapid rise in $I_{1}$ was observed, followed by a decline in current amplitude during sustained application (Fig. 3D). A rapid rise in current was also observed during initial application of $1 \mu \mathrm{M} \mathrm{Az10606120} \mathrm{and}$ $100 \mu \mathrm{M}$ BzATP, but of much smaller amplitude (Fig. $3 E$, $G$, first applications). In all three cases, the subsequent application of 100 $\mu \mathrm{M}$ BzATP alone for $40 \mathrm{~s}$ resulted in the generation of typical biphasic current responses (Fig. $3 D, E, G$ ). In cells responding to $100 \mu \mathrm{M}$ BzATP with biphasic currents, $1 \mu \mathrm{M}$ Az10606120 rapidly abolished $I_{2}$, but only partially inhibited $I_{1}$ (Fig. $3 G$, third application). However, when applied at a $10 \mu \mathrm{M}$ concentration Az10606120 abolished both $I_{1}$ and $I_{2}$ currents in the presence of $100 \mu \mathrm{M}$ BzATP (Fig. $3 F$ ), with a rate highly comparable to that observed after washout of agonist (Fig. 3E, second application). Thus, Az10606120 can prevent development of $I_{2}$ or both currents, depending on the mode of application, but does not alter the development of biphasic currents during subsequent agonist applications. Furthermore, Az10606120 can abolish both currents completely.

\section{Sensitization of receptors}

Figure 3, $E$ and $G$, also shows that the peak amplitude of currents generated by subsequent application of a mixture of $1 \mu \mathrm{M}$ Az10606120 and $100 \mu \mathrm{M}$ BzATP was much higher than during initial application, raising the possibility that P2X7R shows run-up of current, in contrast to other P2XRs, which show rundown of current during repetitive agonist application caused by incomplete recovery from desensitization (North, 2002). To exclude the possibility that run-up of current reflects an artifact caused by incomplete washout of Az10606120, in further experiments we repeatedly stimulated receptors with BzATP only.

In a fraction of cells that responded to initial application of 10 $\mu \mathrm{M}$ BzATP with biphasic currents, repetitive $40 \mathrm{~s}$ applications of $10 \mu \mathrm{M}$ BzATP, with washout periods between agonist applications of $5 \mathrm{~min}$, caused a progressive increase in the peak amplitude of $I_{1}$ and $I_{2}$ (Fig. $4 \mathrm{~A}$ ). During repetitive application of $32 \mu \mathrm{M}$ 
A

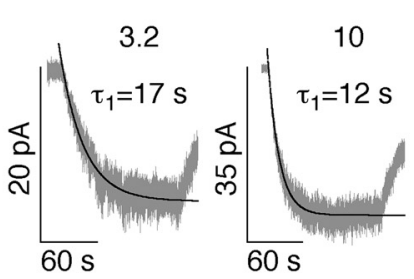

\section{$\operatorname{BzATP}(\mu \mathrm{M})$ :}

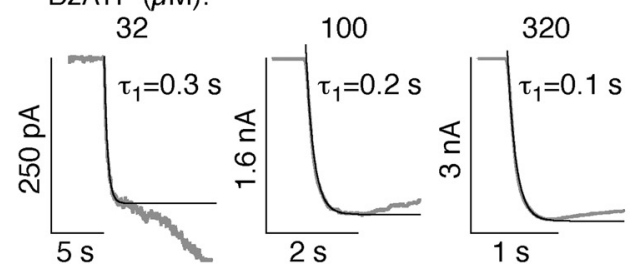

B

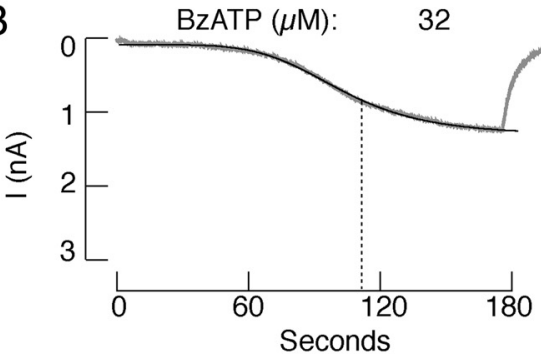

50

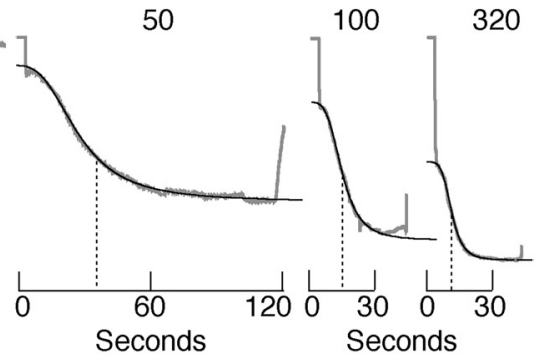

C

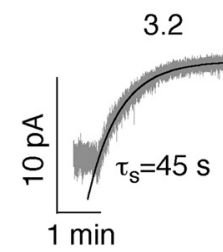

D

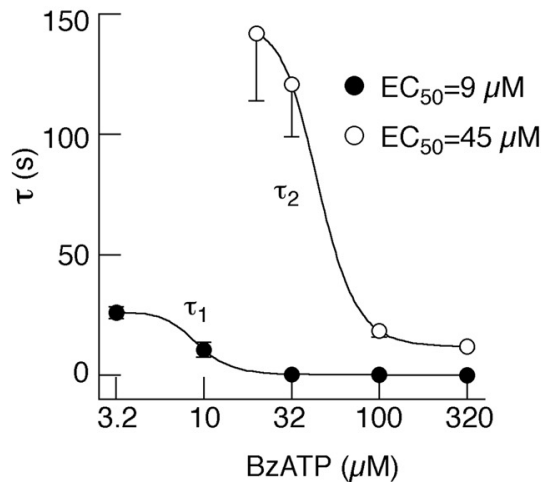

$\operatorname{BzATP}(\mu \mathrm{M})$ :
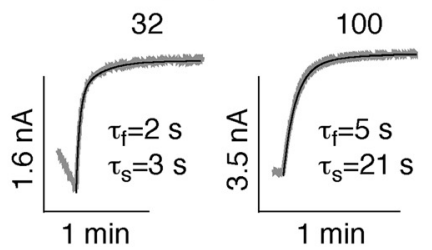

$\mathrm{E}$

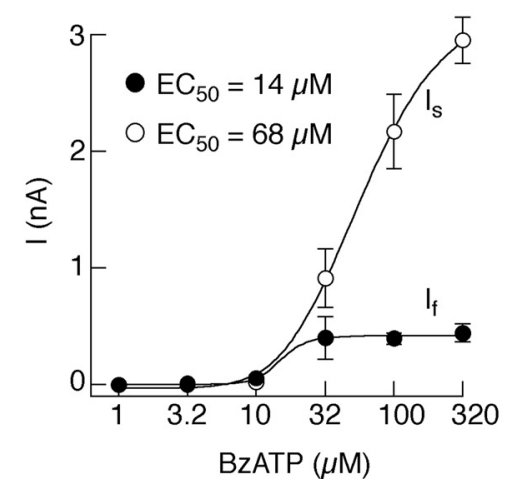

Figure 2. Activation kinetics of P2X7R. A, Single exponential fitting of the $I_{1}$ activation phase. Notice a progressive decrease in the time constant $\left(\tau_{1}\right)$ derived from monoexponential fittings with elevation in BzATP concentration. $\boldsymbol{B}$, The four parameters logistic curve fitting of the $I_{2}$ rising phase. Vertical dotted lines indicate the calculated half-time constants $\left(\tau_{2}\right)$. $C$, Mono- and biexponential fitting of deactivation of P2X7R after washout of BzATP. $\tau_{\mathrm{f}}$ fast deactivation time constants; $\tau_{\mathrm{s}}$ slow deactivation time constants. In $\boldsymbol{A}-\boldsymbol{C}$, representative traces are shown; gray traces indicate experimental records and black lines the fitted curves. $\boldsymbol{D}, \boldsymbol{E}$, Concentration-dependent effects of BzATP on the activation time constants $(\boldsymbol{D})$ and on the $I_{\mathrm{s}}$ and $I_{\mathrm{f}}$ amplitudes derived from deactivation fittings $(\boldsymbol{E})$; mean \pm SEM values from at least 5 records per dose.

BzATP for $40 \mathrm{~s}$ with 5 min washing periods, the amplitude of $I_{1}$ and $I_{2}$ also proportionally increased during the second application, but during the third application the biphasic response was replaced by a monophasic response, with peak current amplitude comparable to that reached at the end of the second agonist application (Fig. 4B). In a case of repetitive application of $100 \mu \mathrm{M}$ BzATP with 5 min washing periods, transition from biphasic to monophasic signaling was achieved after first agonist application (Fig. 4C). In all experiments, increase in the $I_{1}$ amplitude during repetitive agonist application was accompanied by slower receptor deactivation. However, when the steady-state current was reached during repetitive application, there was no further change in the kinetics of receptor deactivation (Fig. $4 B, C$ ). A dependence of the rate of receptor deactivation on duration of

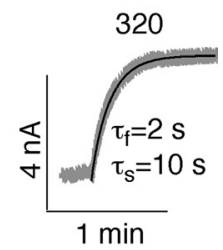

agonist application was also detected; receptors deactivated faster after $1 \mathrm{~s}$ than 40 s agonist application (Fig. $4 D$ ). A train of brief pulses of BzATP was sufficient to cause progressive slowing of deactivation (Fig. 4E).

Once established by stimulation with high $(100$ and $320 \mu \mathrm{M})$ BzATP concentrations, monophasic signaling persisted, even when lower agonist concentrations were subsequently applied. Figure $4 F$ shows the lack of the $I_{2}$ component in cells initially stimulated with $320 \mu \mathrm{M}$ BzATP, followed by subsequent application of 100,32 , and $10 \mu \mathrm{M}$ BzATP, each for $40 \mathrm{~s}$. Monophasic responses were also observed when cells were stimulated initially with $320 \mu \mathrm{M}$ BzATP, followed by $3.2,10,32$, 100 and $320 \mu \mathrm{M}$ BzATP application (data not shown). The calculated $\mathrm{EC}_{50}$ for cells initially stimulated by $320 \mu \mathrm{M}$ BzATP was $24 \pm 0.4 \mu \mathrm{M}(n=4)$, whereas the $\mathrm{EC}_{50}$ for cells initially stimulated with 3.2 , followed by $10,32,100$ and $320 \mu \mathrm{M}$ BzATP application was $64 \pm 3 \mu \mathrm{M}(n=5)$, indicating a significant increase in the potency of BzATP for receptors responding with monophasic currents $(p<0.001)$. Combined with slower deactivation of receptors caused by repetitive agonist application, these results indicate that the receptor sensitizes when repeatedly stimulated.

In further experiments, we studied how long receptors stay in the sensitized state. To exclude the impact of prolonged whole-cell recording, cells were stimulated with $100 \mu \mathrm{M}$ BzATP for $40 \mathrm{~s}$ without recording (Fig. 4G, left), agonist was washed out, the whole-cell recording was established 10-30 min after the washout of agonist, and cells were again stimulated with $100 \mu \mathrm{M}$ BzATP for $40 \mathrm{~s}$ (Fig. 4G, right). When the washout period was 10 min (Fig. 4G, top) and 20 min (data not shown), cells responded to BzATP application with high amplitude monophasic currents, comparable to those observed in the experiments shown in Figure $4 F$. However, when the washout period was $30 \mathrm{~min}$, cells consistently responded to secondary agonist application with typical biphasic currents (Fig. $4 G$, bottom), suggesting the recovery of receptor gating to the unsensitized state. Together, the results of this subsection show that repeated or high dose stimulation locks receptors into a high-affinity, high-conductance state for a prolonged period.

\section{The lack of effect of $\mathrm{Na}^{+}$and $\mathrm{Ca}^{2+}$ influx on sensitization of receptors}

A transition from biphasic to high amplitude monophasic responses during repetitive application of $100 \mu \mathrm{M}$ BzATP for $40 \mathrm{~s}$ was always ( 16 of 16 cells) observed in naive cells bathed in KR buffer (Fig. 5A). To study the relevance of $\mathrm{Na}^{+}$influx in the pattern of signaling, we replaced $90 \%$ (Fig. 5B) or $100 \%$ (Fig. 5C) 
A

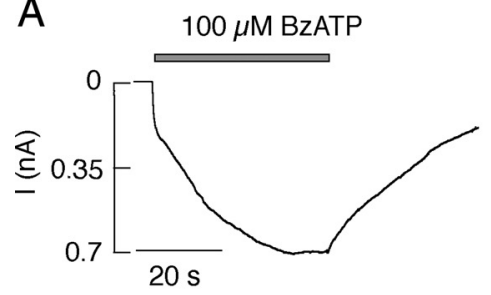

D

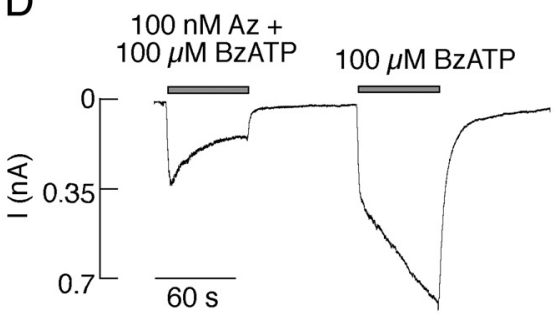

F

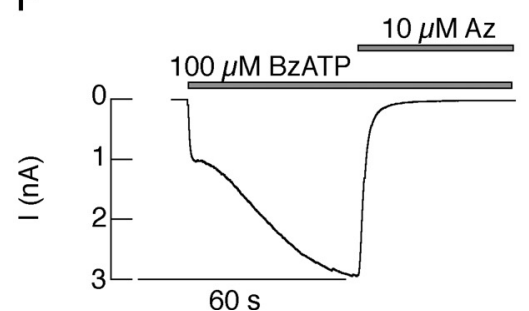

B

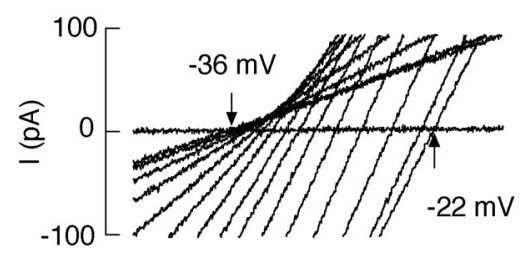

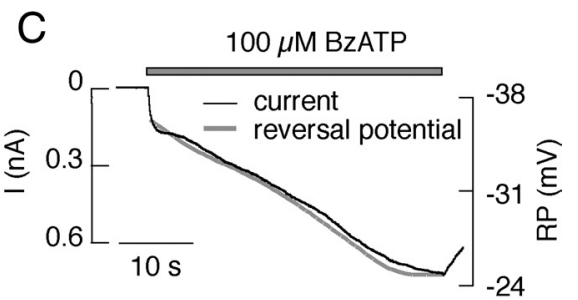

E

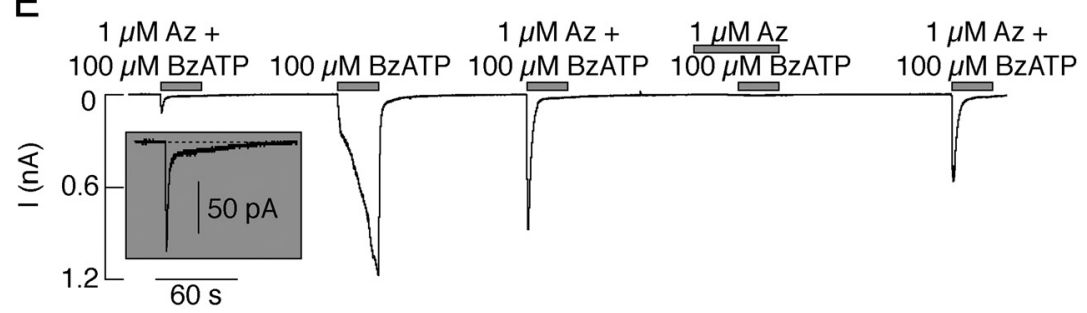

G

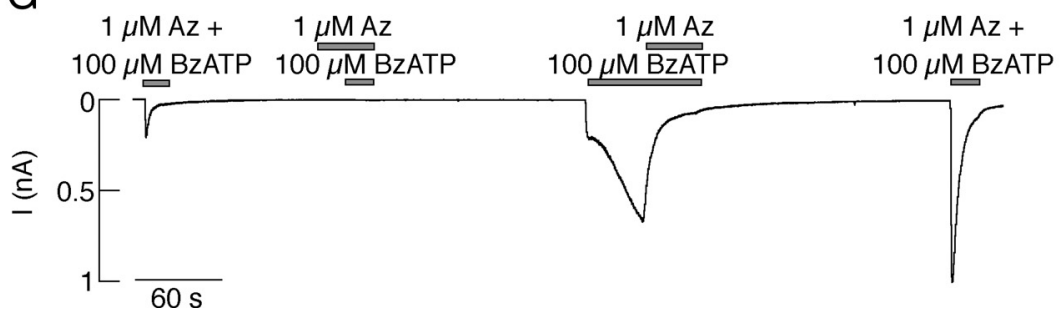

Figure 3. Effects of substitution of bath sodium with NMDG and addition of a P2X7R-specific antagonist on the pattern of agonist-induced current responses. $A-C$, Permeability of the P2X7R pore to NMDG during the sustained agonist application. Pattern of $100 \mu \mathrm{m}$ BzATP-induced current $(\boldsymbol{A})$ and reversal potential ( $\boldsymbol{B}$ ) in cells bathed in sodium-deficient/NMDG-containing Krebs-Ringer buffer during the initial agonist application. Voltage-ramps from $-80 \mathrm{mV}$ to $+80 \mathrm{mV}$ twice per second during $40 \mathrm{~s}$ application were used to generate voltage-current curves. Only selected traces are shown. C, Comparison of kinetics of the secondary current growth (black trace) and changes in the reversal potential (RP, gray trace) during 40 s application of BzATP. $D-G$, Concentration-dependent effect of Az10606120 (Az) on $100 \mu \mathrm{m}$ BzATP-induced current response in cells bathed in Krebs-Ringer buffer. D, Time course of activation and deactivation of receptors in the presence (left) and absence (right) of $100 \mathrm{~nm}$ Az10606120.E, G, Repetitive stimulation of cells with $100 \mu \mathrm{m}$ BzATP in the presence and absence of $1 \mu \mathrm{m}$ Az10606120. Inset shows the magnified first current response; notice the presence of the residual current during sustained application of agonist and antagonist. $\boldsymbol{F}$, Abolition of both $I_{1}$ and $I_{2}$ by $10 \mu \mathrm{m}$ Az10606120, with kinetics comparable to that observed during the washout of agonist ( $\boldsymbol{E}$, second application). In all panels, horizontal bars indicate duration of treatments.

of bath $\mathrm{Na}^{+}$with NMDG. In both experimental conditions, cells responded with typical biphasic currents during the initial agonist application, but the current amplitude decreased with decrease in bath $\mathrm{Na}^{+}$concentration. During the secondary agonist application, all cells responded with monophasic currents, indicating that bath $\mathrm{Na}^{+}$is not essential for the generation of biphasic currents and transition from biphasic to monophasic signaling.

The possible role of $\mathrm{Ca}^{2+}$ influx in generation of biphasic response was examined using several experimental approaches. In one series of experiments, cells were bathed in $\mathrm{Ca}^{2+}$-deficient $\mathrm{KR}$ buffer and the patch pipette was filled with $\mathrm{Ca}^{2+}$-deficient medium supplemented with $\mathrm{Ca}^{2+}$ chelating agents, 10 mM EGTA (Fig. $5 D$ ) or 5 mm BAPTA (Fig. 5E). This should effectively buffer fluctuations in the intracellular $\mathrm{Ca}^{2+}$ concentration coming from the influx of residual $\mathrm{Ca}^{2+}$ present in the bath medium. Under such experimental conditions, generation of biphasic currents during initial agonist application and a shift from biphasic to high amplitude monophasic responses were also observed. To exclude the possible side effect of reduction in divalent cation concentrations in the bath medium, in a second set of experiments, $\mathrm{Ca}^{2+}$ was substituted with $\mathrm{Mg}^{2+}$. In cells clamped at -60 $\mathrm{mV}$, a transition from biphasic to monophasic currents was also observed (Fig. 5F). Finally, to decrease the electrochemical gradient for $\mathrm{Ca}^{2+}$, cells bathed in $\mathrm{Ca}^{2+}$-deficient medium with 10 $\mathrm{mm}$ EGTA in the pipette medium were held at $+60 \mathrm{mV}$ during repetitive agonist application. Still, the transition from biphasic to high amplitude monophasic response occurred during repet- itive application of $10 \mu \mathrm{M}$ BzATP (Fig. 5G). Receptor deactivation was also slowed during secondary application of BzATP in cells bathed in $\mathrm{Ca}^{2+}$-deficient bath medium with pipette medium containing no added $\mathrm{Ca}^{2+}$ and having 10 mM EGTA (Fig. $5 H$, left vs right). Thus, bath $\mathrm{Ca}^{2+}$ is also not required for the transition from biphasic to monophasic signaling.

Dependence of receptor gating on the $\mathrm{N}$-terminal structure We reported recently that several N-terminal mutants have altered pore dilation (Yan et al., 2008). Figure $6 A-C$, left, shows the $I-V$ relationship of cells under the ramp voltage protocol during the initial agonist application in three such mutants. Like the wild-type receptor, the K17A mutant responded with a gradual shift in the reversal potential when cells were bathed in NMDGcontaining KR buffer. In contrast, the T15K and T15E mutants showed immediate permeability to NMDG during the initial agonist application and no additional changes in the reversal potential during $40 \mathrm{~s}$ agonist application. Here, we used these mutants to study the dependence of receptor deactivation on the status of the channel pore. Deactivation kinetics was examined in cells exposed to BzATP for $1 \mathrm{~s}$ (Fig. 6A-C, middle left traces) and $2 \times 40 \mathrm{~s}$ with $10 \mathrm{~min}$ washout period (Figs. $6 A-C$, middle right and right traces). The K17A mutant deactivated relatively rapidly after $1 \mathrm{~s}$ stimulation and the participation of the $\tau_{\mathrm{s}}$ deactivation time constant was minor, whereas the T15K and T15E mutants deactivated slowly. Like the wild-type receptor, the K17A mutant also showed a transition from biphasic to monophasic currents 

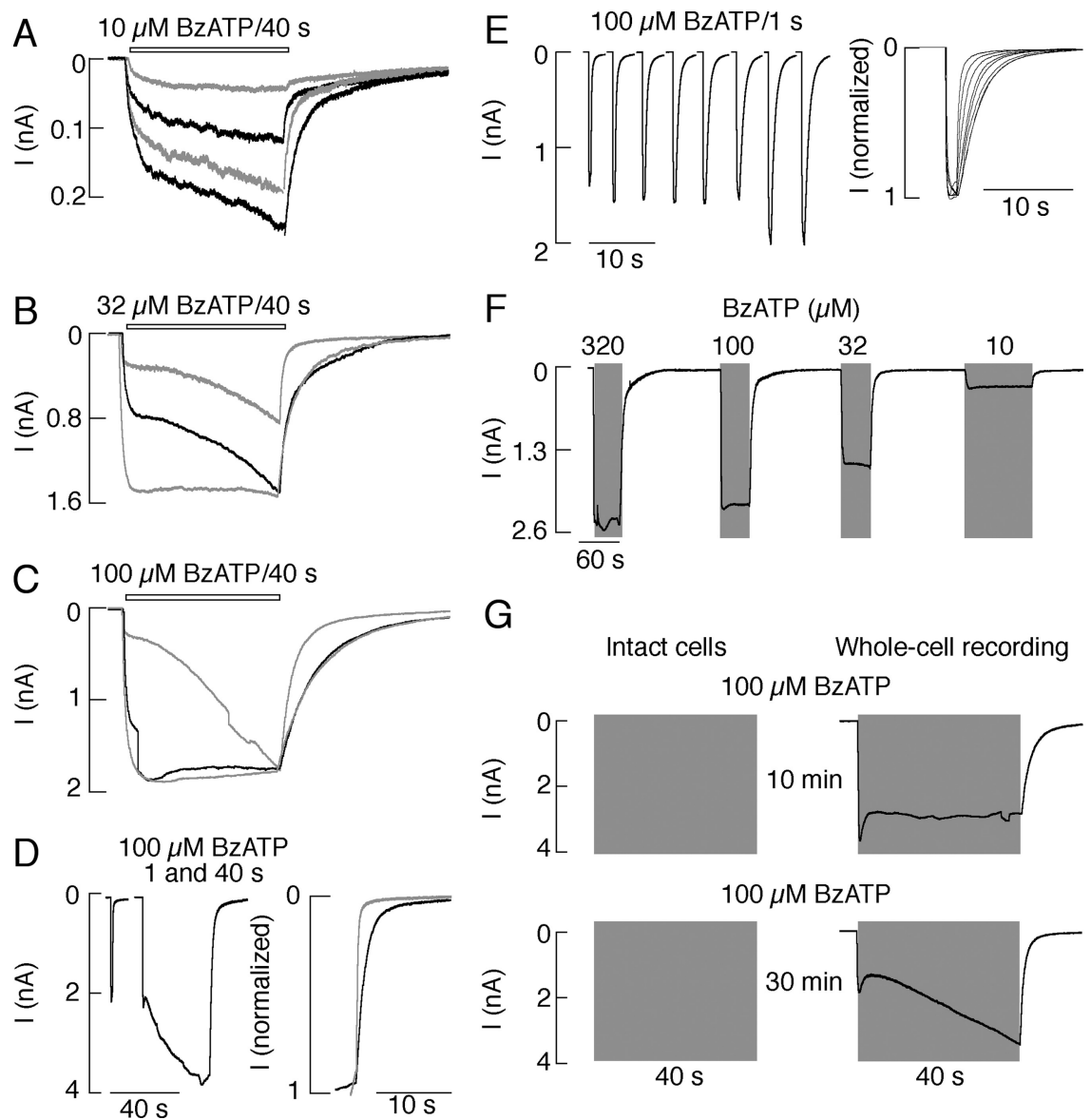

Figure 4. Receptors sensitize during repetitive agonist application. $A-C$, Time-dependent increase in the peak amplitude of current and a decrease in the rate of receptor deactivation in cells stimulated repeatedly with $10 \mu \mathrm{m}(\boldsymbol{A}) 32 \mu \mathrm{m}(\boldsymbol{B})$, and $100 \mu \mathrm{m}(\boldsymbol{C})$ BzATP for $40 \mathrm{~s}$. For clarity, every second trace is shown as gray. $\boldsymbol{D}$, A difference in the rate of receptor deactivation caused by short (1 s) and prolonged (40 s) stimulation with BzATP. E, A decrease in the rate of receptor deactivation was induced by repetitive short (1 s) application of BzATP followed by 2 min washout periods. $\boldsymbol{F}$, Sensitized receptors respond with monophasic currents during the subsequent application of BzATP, with the peak amplitude of current determined by agonist concentration. $\mathbf{G}$, Duration of the sensitized state after the washout of agonist. Intact cells ready to be patched were initially stimulated with $100 \mu \mathrm{m}$ BzATP for $40 \mathrm{~s}$ (left) followed by washout period of variable times. Before the secondary agonist application, the whole-cell patch clamp was established and current response was recorded. The response of cells after the washout period of $10 \mathrm{~min}$ (top) and $30 \mathrm{~min}$ (bottom) are shown.

during repetitive application of BzATP, accompanied with slower receptor deactivation. In contrast, $\mathrm{T} 15 \mathrm{~K}$ and T15E mutants responded to a second agonist application with a pattern of current and rates of deactivation highly comparable to those observed during the initial agonist application. The T15E mutants showed higher sensitivity to BzATP compared with the wild-type receptor; the estimated $\mathrm{EC}_{50}$ values for BzATP were: $64 \pm 3 \mu \mathrm{M}$ $(n=5)$ for the wild-type receptor and $2 \pm 0.1 \mu \mathrm{M}(n=4)$ for the T15E mutant. Figure $6 D$ shows that the mutant always responded with monophasic currents to initial application of BzATP in 1-100 $\mu \mathrm{M}$ concentration range, with rates of activation increasing with agonist concentrations. Thus, whether the sensitized state is induced by preexposure to ATP in wild-type cells or is permanently present in mutants, large-amplitude monophasic currents are always coupled to slow deactivation.

\section{Simulations with the P2X7R Markov state gating model}

The responses of P2X7R to single applications of BzATP to naive cells at increasing concentrations illustrated in Figure 1 can be reproduced using the Markov state model consisting of 8 States described by the scheme in Figure 7 and Equations 1-9. Each state in this scheme represents the fraction of receptors that are bound to at most three agonist (BzATP) molecules.

The structure of the scheme shown in Figure 7 can be interpreted as follows. Receptors in the $C_{1}$ and $C_{4}$ states have no BzATP bound, receptors in states $C_{2}$ and $C_{3}$ have one BzATP bound, receptors in $Q_{1}$ and $Q_{4}$ states have two BzATP bound and receptors in states $Q_{2}$ and $Q_{3}$ have three BzATP bound. The states $C_{\mathrm{i}}(i=1$, $2,3,4)$ correspond to receptors possessing closed channel pores, whereas those in states $Q_{\mathrm{i}}(i=1,2,3,4)$ have open pores. The states in the top row $\left(C_{1}, C_{2}, Q_{1}\right.$ and $Q_{2}$ ) represent unsensitized receptors, which are interpreted as having undilated channel pores with relatively small conductance and relatively negative reversal potential, given a set of mixed ionic concentrations in the medium. Naive channels (channels not previously exposed to agonist) are assumed to be unsensitized. The states in the bottom row $\left(C_{3}, C_{4}, Q_{3}\right.$ and $Q_{4}$ ) represent receptors that are sensitized, having dilated pores with larger conductance and less negative reversal potentials when tested in NMDG-containing medium; in the simulations shown, the reversal potentials are the same for all states because the medium contained almost exclusively $\mathrm{Na}^{+}$.

Here we assume that ligand binding entails negative cooperativity. Naive and unsensitized receptors (state $C_{1}$ ) are assumed to be symmetric, with the three binding sites having equal and high affinity for binding BzATP (i.e., $3 k_{2} / k_{1}$ is relatively large), but the occupation of one binding site by BzATP leads to the loss of symmetry and an assumed reduction in the affinity of the two remaining binding sites due to a conformational change (i.e., $\left.k_{4} / k_{3}<3 k_{2} / k_{1}\right)$. When a second site is occupied, the affinity of the remaining binding site is further reduced $\left(k_{6} /\left(3 k_{5}\right)<k_{4} / k_{3}\right)$. When all binding sites are occupied, however, symmetry is assumed to be restored, and the binding rates revert to those of a naked receptor. A slow conformational change is assumed for the fully occupied receptor, leading to a slow dilation of the pore (transition rate $L_{3}$ from $Q_{2}$ to $Q_{3}$ ). (A possible interpretation is that restored symmetry with all sites bound relieves a mechanical stress, which permits other processes that mediate dilation to take effect. Note, however, that the performance of the model depends only on the numerical values assigned to the parameters, not on the interpretations that motivated the assignments.) The restoration of symmetry is also assumed to return the binding and unbinding rates to those of an unbound channel $\left(k_{2}\right.$ and $\left.k_{1}\right)$ and to persist as ligand unbinds and rebinds within the sensitized regime; this last assumption allows the model to reproduce the fast, high affinity openings to a dilated state that are observed once sensitization is established. Deactivation of sensitized receptors is also much slower than that of unsensitized receptors because $2 k_{1} \ll 2 k_{3}, 3 k_{5}$. The smallness of the rate of the back transition from sensitized to unsensitized states (rate $L_{2}$ from $Q_{3}$ to $Q_{2}$ ) causes the receptor to act as a 


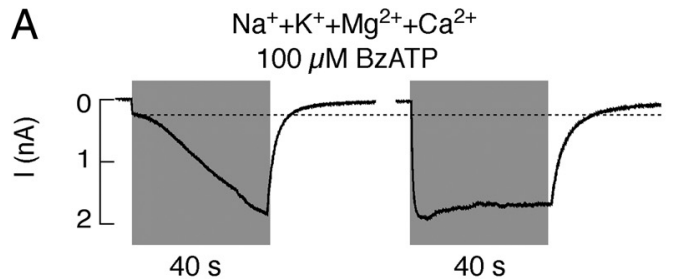

B
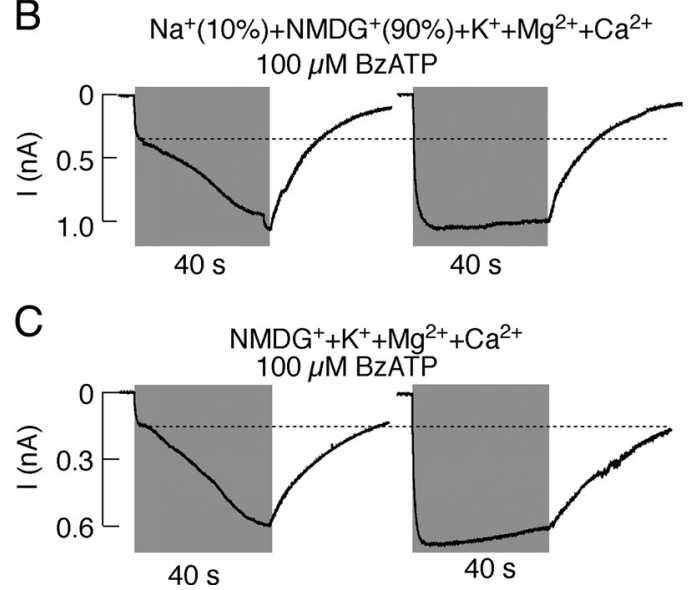

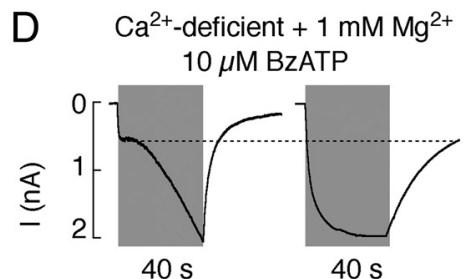

E $\quad \mathrm{Ca}^{2+}$-deficient $+1 \mathrm{mM} \mathrm{Mg}^{2+}$

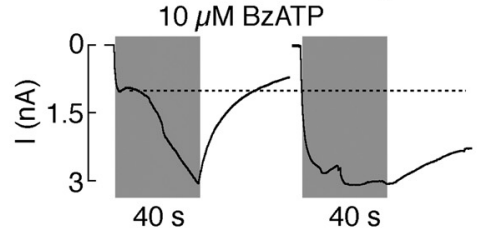

$\mathrm{H}$

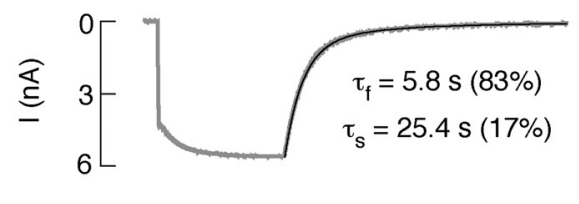

$\mathrm{Ca}^{2+}$-deficient $+1 \mathrm{mM} \mathrm{Mg}^{2+}$

\section{F $\quad \mathrm{Ca}^{2+}$-deficient $+3 \mathrm{mM} \mathrm{Mg}^{2+}$ $100 \mu \mathrm{M}$ BzATP}

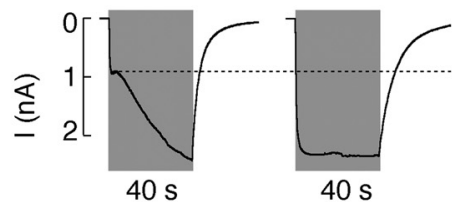

G $\mathrm{Ca}^{2+}$-deficient + $1 \mathrm{mM} \mathrm{Mg}^{2+}$

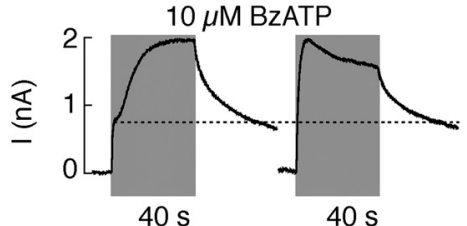

$40 \mathrm{~s}$ $100 \mu \mathrm{M}$ BzATP/40s

Figure 5. Sensitization of receptors is independent of bath sodium and calcium. $\boldsymbol{A}-\boldsymbol{C}$, Cells were bathed in KR buffer containing Na ${ }^{+}, \mathrm{K}^{+}, \mathrm{Mg}^{2+}$ and Ca ${ }^{2+}(\boldsymbol{A})$, and in KR buffer in which $90 \%$ (B) or $100 \%(C)$ of $\mathrm{Na}^{+}$was replaced by NMDG, with a pipette solution containing $10 \mathrm{~mm}$ EGTA. Left, Biphasic responses observed during the initial agonist application. Right, High amplitude monophasic responses were always observed during the second application of $100 \mu \mathrm{m}$ BzATP after 2 min washout periods. $\boldsymbol{D}, \boldsymbol{E}$, Transition from biphasic to high amplitude monophasic current responses was observed in cells bathed in $\mathrm{Ca}^{2+}$-deficient KR buffer with the pipette medium containing $10 \mathrm{~mm}$ EGTA (D) or $5 \mathrm{~mm} \mathrm{BAPTA} \mathrm{(E).} \mathrm{In} \mathrm{both} \mathrm{experiments} \mathrm{cells} \mathrm{were} \mathrm{clamped} \mathrm{at}-60 \mathrm{mV}$. $\boldsymbol{F}$, $\boldsymbol{G}$, Sensitization of receptors was also observed in cells bathed in medium in which $\mathrm{Ca}^{2+}$ was substituted with $\mathrm{Mg}^{2+}(\boldsymbol{F})$, as well as in cells containing $1 \mathrm{~mm} \mathrm{Mg}{ }^{2+}$ and clamped at $+60 \mathrm{mV}(\boldsymbol{G})$. In both experiments, the pipette medium contained $10 \mathrm{~mm}$ EGTA. $\boldsymbol{H}, \mathrm{In} \mathrm{Ca}^{2+}$-deficient medium and with pipette medium containing $10 \mathrm{~mm}$ EGTA, transition from biphasic to high amplitude monophasic currents was also accompanied with slower rate of deactivation. Because of the drop in the divalent cation concentrations in experiments shown in $\boldsymbol{D}, \boldsymbol{E}$, and $\mathbf{G}, 10 \mu \mathrm{m}$ BzATP was used.

ratchet, accumulating memory of ATP exposure in the form of persistent sensitization. The slowest rate is the loss of sensitization (return from $C_{4}$ to $\left.C_{1}\right)$ during washout $\left(L_{1} \ll L_{2}, L_{3}\right)$.

Using this scheme, we successfully generate the expected monophasic response to 3.2 and $10 \mu \mathrm{M}$ BzATP and the biphasic response to 32, 100 and $320 \mu \mathrm{M}$ BzATP, as shown in Figure 8A. In the spirit of parsimony we have, with one exception, used the same parameter values (listed in Table 1) for all simulations rather than search for the best fit to each trace. The exception is that we have set $L_{3}$ to $0.1 \mathrm{~s}^{-1}$ for the case of $10 \mu \mathrm{M}$ BzATP; if the value of $L_{3}$ is kept at $0.5 \mathrm{~s}^{-1}$, the response is biphasic. In fact, biphasic responses are sometimes seen at $10 \mu \mathrm{M}$ (data not shown), so this may reflect heterogeneity among cells. The deactivation component of the current exhibited, in most cases, the biphasic response observed experimentally, but the slow monophasic response was less obvious due to the relatively large value of $k_{1}$. Decreasing the value of $k_{1}$ would generate slow, monophasic deactivation for single agonist presentations, but other features of the model (including the slowing down of the deactivation component during repetitive stimulation shown below) would be lost. The use here of one parameter set to describe all features limits the ability of the model to capture all the behaviors of the heterogeneous population of cells used to generate the experimental current recordings.

The model also successfully displayed the initial dominance of the slow current $I_{2}$ over the fast current $I_{1}$ in moderate $40 \mathrm{~s}$ BzATP stimulation (Fig. $8 B$ ) seen in the experiments (Fig. 1). Here, guided by the indication above that cells are heterogeneous, we constructed a population of 10 model cells by randomly selecting model parameters (except for $L_{3}$ ) using the normal distribution with SDs listed in Table 1 and using the uniform distribution to select random values for $L_{3}$ from a numerically estimated range specified in Table 1. When higher concentrations of BzATP were applied, $I_{1}$ dominated $I_{2}$. In agreement with the experiments shown in Figure 1, the simulated $I_{1}$ current showed a monodirectional dependence on the concentration of BzATP, as opposed to the bidirectional dependence of $I_{2}$ in the same range of BzATP concentration. The total increase in current $\left(I_{1}+I_{2}\right)$ as a function of BzATP concentration, averaged over the 10 cells, showed a very similar dose-response curve to that obtained in Figure $1 C$ with comparable $\mathrm{EC}_{50}(45 \mu \mathrm{M}$; Fig. $8 \mathrm{~B}$, inset $)$.

Examination of the occupancy of the individual channel states, however, allows us to interpret the meaning of $I_{1}$ and $I_{2}$ more precisely in the model. Figure $8 \mathrm{C}$ shows the states in response to $32 \mu \mathrm{M}$ BzATP. The fast $I_{1}$ component corresponds to channels moving from state $C_{1}$ through $C_{2}$ to $Q_{1}$ or $Q_{2}$, whereas the slow $I_{2}$ component corresponds to channels moving from $Q_{2}$ to $Q_{3}$. When the agonist is removed, the channels that were in $Q_{3}$ or $Q_{4}$ go to $C_{4}$ rather than $C_{1}$, and this accounts for the slow component of deactivation, as the rate of deactivation via $k_{1}$ is much slower than deactivation via $k_{3}$ or $k_{5}$. Figure $8 D$ shows the states in response to $320 \mu \mathrm{M}$. At this higher agonist concentration, channels move relatively quickly from $C_{1}$ to $Q_{3}$, passing through $Q_{1}$ and $Q_{2}$ only transiently, the current appears monophasic, and only the $I_{1}$ component is evident in Figure $8 \mathrm{~A}$. The model thus suggests that if current components are interpreted in terms of channel occupancy rather than rate of rise, the $I_{1}$ component at high agonist concentration corresponds to the sensitized states, whereas the $I_{1}$ component at lower concentrations is generated by the unsensitized states.

The model can also account for the responses to repetitive agonist application that were illustrated in Figure 3. Simulating these experiments imposes more constraints on the model than simulating the single presentations alone. As shown in Figure 9A, 
A

K17A

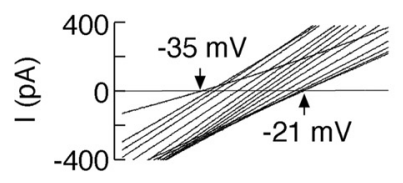

B

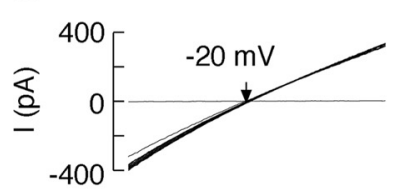

C

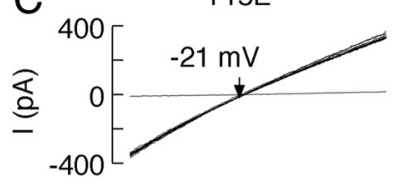

D
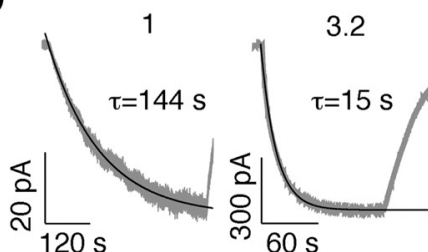

3.2
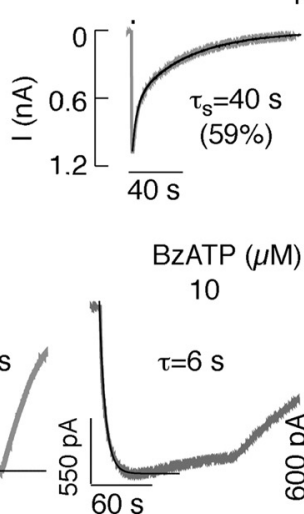

$100 \mu \mathrm{M}$ BzATP
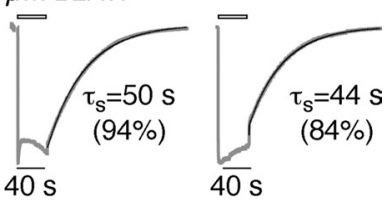

$100 \mu \mathrm{M}$ BzATP

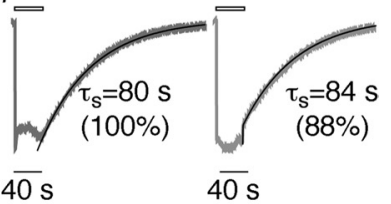

$40 \mathrm{~s}$

32

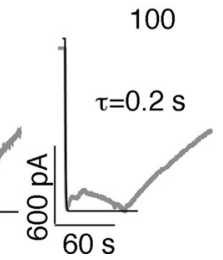

Figure 6. Dependence of P2X7R sensitization on the N-terminal structure. $A-C$, Characterization of the K17A $(A), T 15 K(B)$, and T15E (C) mutants. The T15K and T15E mutants instantaneously opened with the dilated state, as indicated by the value of the reversal potential and the lack of a positive shift during prolonged agonist application. In contrast, the K17A mutant dilates gradually during the initial agonist application, as indicated by the rightward shift in the reversal potential (left). Similar records have been shown previously (Yan et al., 2008). Three mutants differed in their deactivation time kinetics during the short (1 s) and prolonged $(2 \times 40 \mathrm{~s}$ ) stimulation. Notice the slow deactivation of T15K and T15E mutants and the lack of changes in the rate of receptor deactivation after secondary agonist application, in contrast to the K17A mutant. $\boldsymbol{D}$, Concentration-dependent effects of BzATP on the rate of T15E mutant receptor activation, which shows the lack of the secondary current growth in all BzATP concentrations.

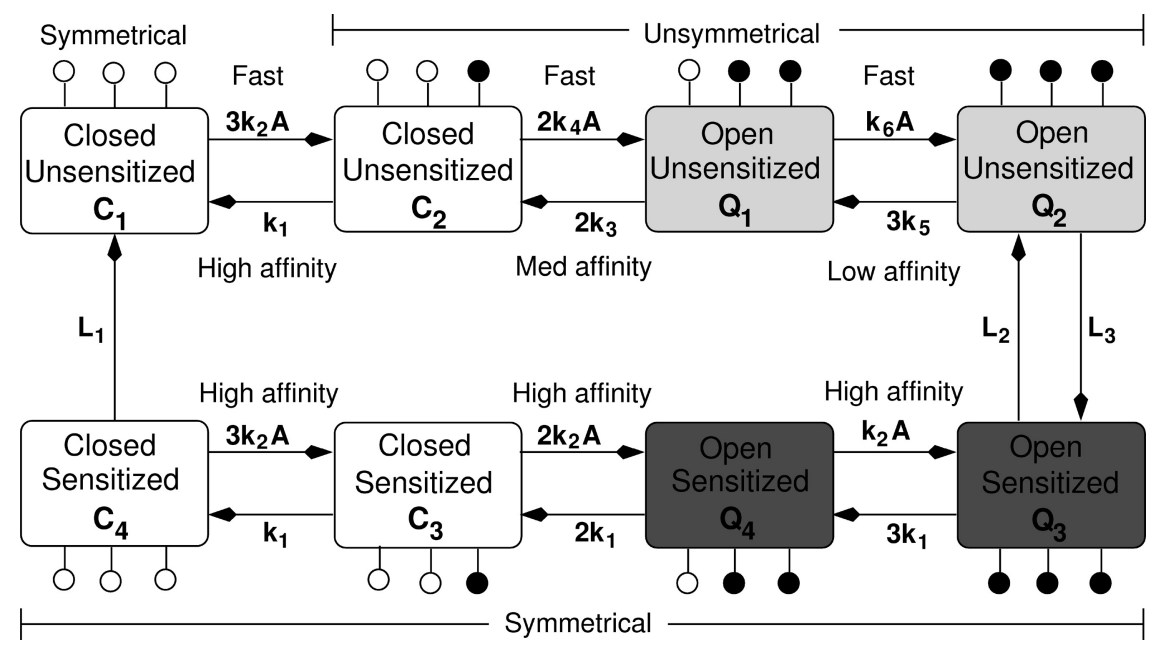

Figure 7. Markov state cell model describing the sequence of binding/unbinding and sensitization/loss-of-sensitization events of P2X7Rs in a cell. The states $C_{i}$ (closed channel pore) and $Q_{i}$ (open channel pore), $i=1,2,3,4$, represent the fraction of receptors in a given state. White (black) circles on each state indicate unoccupied (occupied) binding sites ( 3 in total) on the receptors of that state. The upper row corresponds to the unsensitized (undilated channel pore) states, while the bottom row corresponds to the sensitized states (open ones are dilated). $k_{\mathrm{i}}, i=1,2, \ldots, 6$ and $L_{\mathrm{i}}, i=1,2,3$, are the transition rates between the different states and $A$ is the agonist concentration (BzATP or ATP). The gating variables and reversal potentials of $Q_{1}\left(Q_{3}\right)$ and $Q_{2}\left(Q_{4}\right)$ are the same (Table 1). repeated stimulation of the model cell with $10 \mu \mathrm{M}$ BzATP for $40 \mathrm{~s}$ steadily increased current amplitude and slowed down the deactivation of the current. Examining the states (data not shown) indicates that this is due to the accumulation of a small fraction of receptors in the lower row of Figure 7, which then go to $C_{4}$ instead of $C_{1}$ during washout. When the agonist is reapplied, there are rapid, high affinity openings directly to $Q_{4}$ and $Q_{3}$ without passing through $Q_{1}$ and $Q_{2}$ in addition to the openings from $C_{1}$ that made up the sole contribution in the first pulse. This behavior was modified when the concentration of BzATP was increased to 32 and $100 \mu \mathrm{M}$ in Figure 9, $B$ and $C$, respectively. In these two cases, the current amplitude increased at each agonist application but the slow $I_{2}$ component of the current decreased due to sensitization (i.e., receptor accumulation in the lower row of Fig. 7). Thus, the current reached its maximum amplitude after a few pulses and the fast current $I_{1}$ progressively dominated $I_{2}$. The higher agonist concentrations in $\mathrm{B}$ and $\mathrm{C}$ than in A yield a much larger proportion of channels along the bottom row of sensitized states, progressively reducing the number available to open along the top row. As the channels opening from $C_{4}$ go directly to a dilated state, they do not contribute to the slow $I_{2}$ component, which therefore declines over time as openings along the bottom row become dominant. In all three panels $(A-C)$, current deactivation during washout became slower in parallel to the increase of the $I_{1}$ component as the sensitized pool grew at the expense of the unsensitized pool.

The model also captures the experimental observations shown in Figure $4, D$ and $E$. In $100 \mu \mathrm{M}$ BzATP, a long 40 s pulse produced slower deactivation than a short $1 \mathrm{~s}$ pulse (Fig. $4 D$ ). A train of short pulses separated by $10 \mathrm{~s}$ washout periods resulted in a dramatic slowing of deactivation with only a modest increase in amplitude (Fig. $4 E)$; very similar results were obtained with washout periods up to $20 \mathrm{~min}$, because of the very slow rate of return from $C_{4}$ to $C_{1}$. In Fig. 9, $D$ and $E$, the slowing of deactivation is caused by an accumulation of channels in the sensitized state, which mostly close by going to state $C_{4}$. The observation recorded in Figure $4 F$ showing monophasic currents even at low concentrations of BzATP for 40 s once exposed to $320 \mu \mathrm{M}$ was also exhibited by the model cell (Fig. 9F). The initial pulse brought most of the receptors to the states $Q_{3}$ and $Q_{4}$ so that they closed to state $C_{4}$ when the agonist was removed. The biphasic current re- 

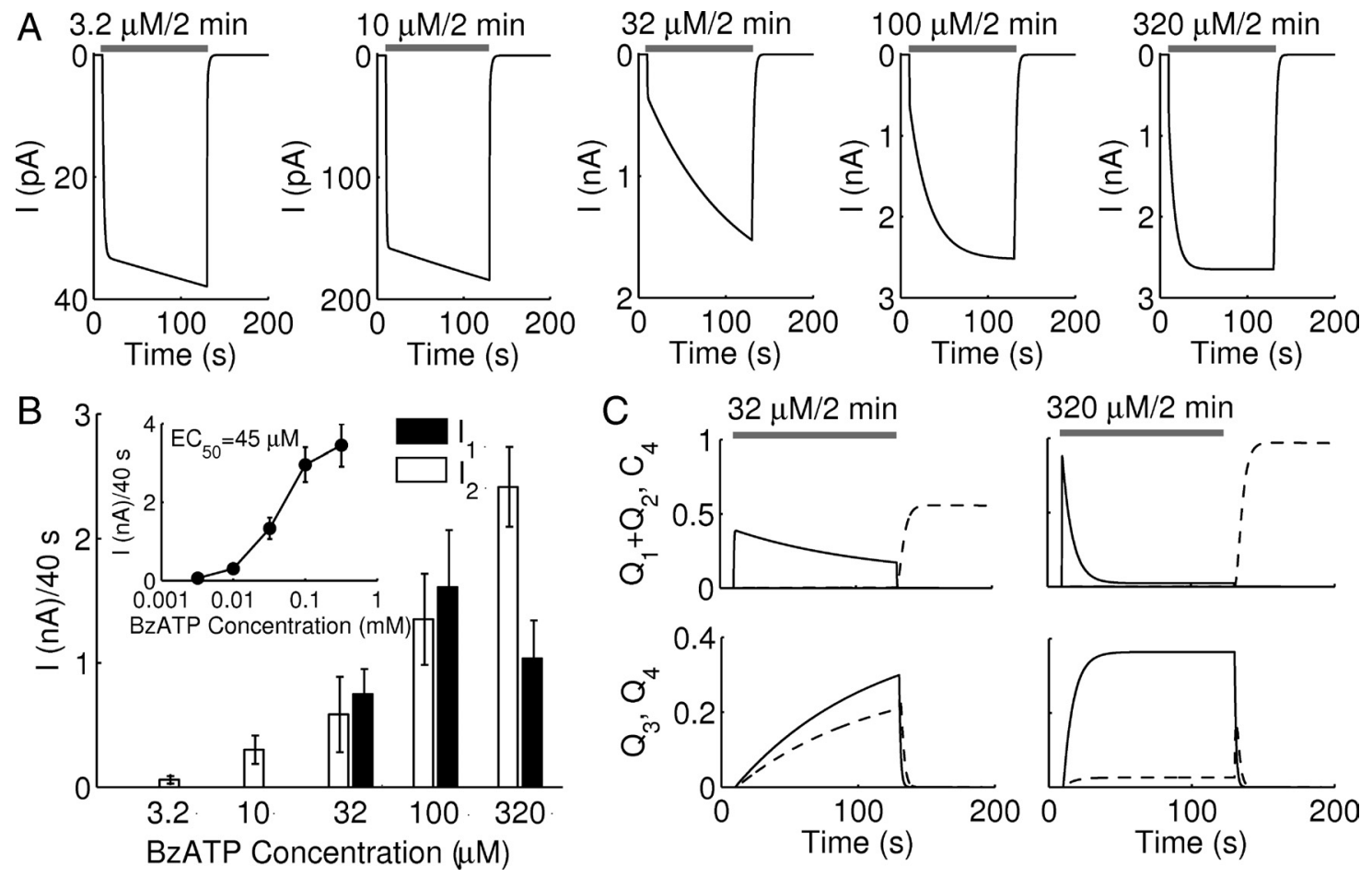

Figure 8. Current responses to increasing doses of agonist concentration according to the Markov state cell model, Equations 1-9. $A$, Current simulations obtained from the cell model upon 3.2, $10,32,100$, and $320 \mu \mathrm{m} / 2 \mathrm{~min}$ BzATP stimulation. $\boldsymbol{B}$, Average amplitudes of the fast, $I_{1}$, and slow, $I_{2}$, currents in response to 40 s agonist (BzATP) stimulation of a heterogeneous population of $n=$ 10 cell models, randomly generated using the normal and uniform distributions according to Table 1 . Notice the monodirectional dose dependence of $I_{1}$ and bidirectional dose dependence of $I_{2}$ on BzATP. Inset, Dose-response curve of the total current $I\left(=I_{1}+I_{2}\right)$ after 40 s stimulation with 3.2, 10,32, 100, and $320 \mu \mathrm{m}$ BzATP; mean \pm SEM values from 10 model cells per dose. The estimated $\mathrm{EC}_{50}$ obtained from these stimulations is $45 \mu \mathrm{m}$. $C, D$, The time evolution of the states $Q_{1}+Q_{2}$ (solid, top), $C_{4}$ (dashed, top), $Q_{3}$ (solid, bottom) and $Q_{4}$ (dashed, bottom) are shown during $40 \mathrm{~s}$ stimulation with $32 \mu \mathrm{M}(\boldsymbol{C})$ and $320 \mu \mathrm{M}(\boldsymbol{D})$ BzATP.

sponse typical of these cells when applying lower doses of BzATP was then lost because the openings proceeded from states $C_{4}$ and $C_{3}$ directly to $Q_{3}$ and $Q_{4}$. Only a long washout of 30 min or longer (data not shown) allows enough time for the receptors to transition back to $C_{1}$ and restore biphasic currents.

All of the responses of the receptors to the P2X7R-specific antagonist Az10606120 shown in Figure 3 were also reproduced by the model under the assumptions that Az10606120 reduces all the forward rate constants $\left(k_{2}, k_{4}\right.$, and $\left.k_{6}\right)$ with a time constant of $15 \mathrm{~s}$ and also that the onset of antagonism was delayed by $1 \mathrm{~s}$ (supplemental Fig. S1, available at www.jneurosci.org as supplemental material). The latter assumption enabled the model to reproduce the initial spikes of current seen when Az10606120 was applied simultaneously with BzATP (Fig. 3E, first and third applications). In contrast, if the antagonist was assumed to apply only to unsensitized receptors, then a high amplitude sustained current carried by sensitized receptors was predicted, independently of the time of antagonist application (supplemental Fig. S2, available at www.jneurosci. org as supplemental material, third, fourth, and fifth application), in contrast to experimental observations (Fig. $3 E, G$ ).

In summary, all the experimental results in Figures 1, 3, and 4 are shown by the model to be explainable as consequences of shifting receptors, to a greater or lesser degree, from the unsensitized state to the sensitized state, with its fast, high affinity openings and slow deactivations.

\section{Discussion}

We have shown here that gating properties of P2X7R depend on agonist concentration and duration of its application and washout periods. Current measurements indicated that naive P2X7R activated and deactivated monophasically at low and biphasically at higher agonist concentrations. We also observed monophasic and biphasic responses in single cell calcium measurements using the low affinity indicator Fura-FF. However, that method is of limited use in studies on gating of P2X7R because of dye leak during sustained agonist application (Yan et al., 2008).

Biphasic current and calcium responses are consistent with the two-pore hypotheses, with the P2X7R pore accounting for $I_{1}$ and activation of the additional pore secondary to receptor activation accounting for $I_{2}$. It also has been suggested that calcium acts as a second messenger for opening the pore associated with P2X7R (Faria et al., 2005). Association of P2X7R with pannexin-1 hemichannels has also been shown and appears to be critical for P2X7R-induced dye uptake and interleukin- $1 \beta$ release (Pelegrin and Surprenant, 2009). Others have proposed the existence of two distinct ATP activation sites (Klapperstück et al., 2001) and suggested that the C-terminal deletion-induced loss of a low affinity binding site accounts for the loss of most of the $I_{1}$ current and complete loss of the $I_{2}$ current and that the residual $I_{1}$ current, whose amplitude resembles monophasic currents detected at low agonist concentration, reflects activation of high affinity binding sites (Becker et al., 2008).

In contrast, here we show that generation of biphasic currents was independent of bath $\mathrm{Ca}^{2+}$ concentration. Generation of biphasic currents is also not blocked by inhibition of pannexin-1 channels, and is also observed in cells not expressing pannexins (Yan et al., 2008), and $I_{2}$ growth temporally correlates with the development of permeability for NMDG. In addition, if the slow growth of $I_{2}$ reflects conductivity of other channels/transporters, the contribution of this component of inward current should increase with increase in agonist concentrations. In contrast, we observed bidirectional changes in $I_{2}$ amplitude with increase in 


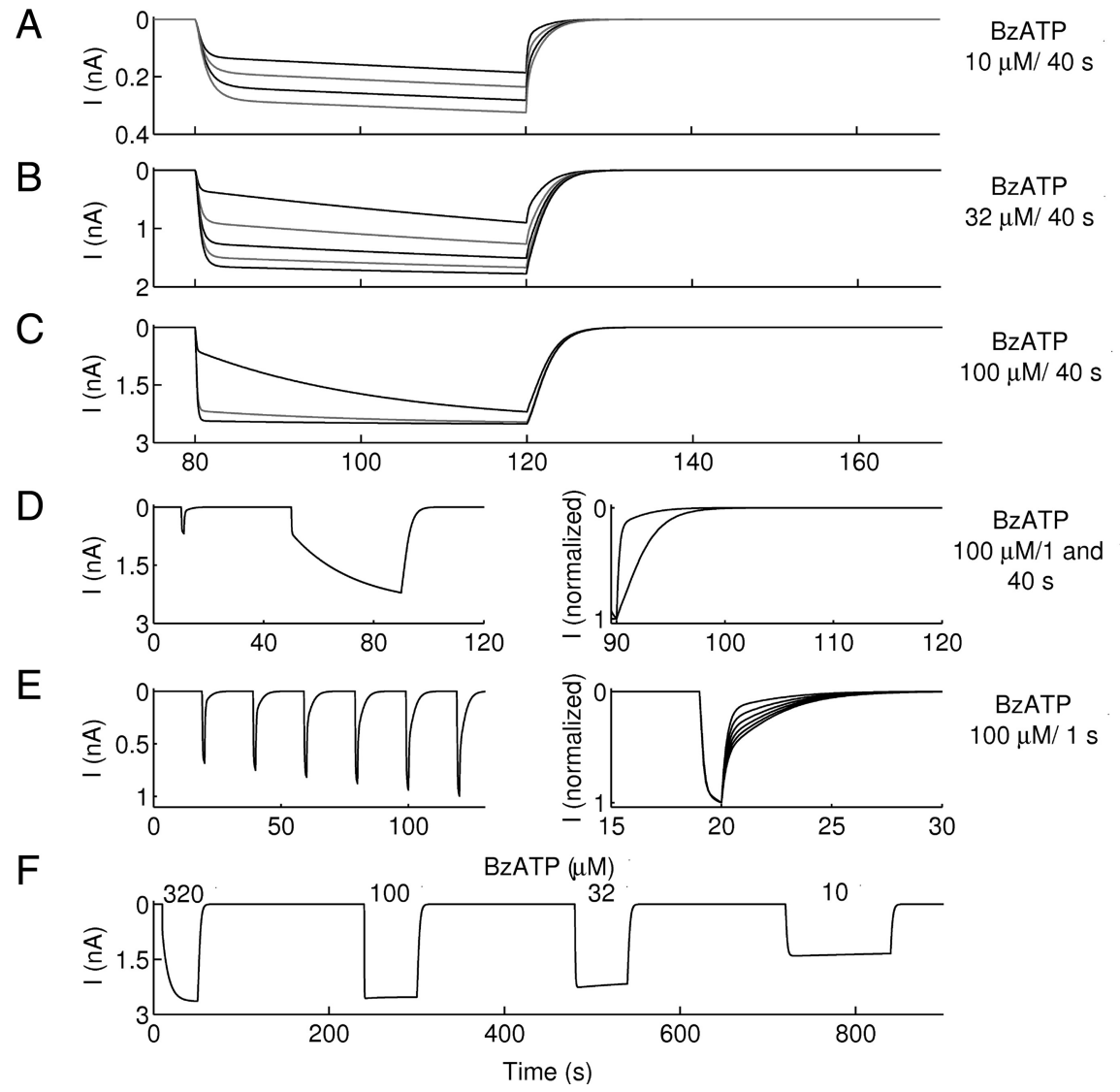

Figure 9. Simulated current responses to repeated application of BzATP using the Markov state cell model and Equation 9. $A-C$ Simulations of periodic stimulation with $10 \mu \mathrm{m} / 40 \mathrm{sBzATP}(\boldsymbol{A}), 32 \mu \mathrm{m} / 40 \mathrm{~s} \operatorname{BzATP}(\boldsymbol{B})$, and $100 \mu \mathrm{m} / 40 \mathrm{sBzATP}(\boldsymbol{C}$. D, Simulation of model cell initially stimulated with $100 \mu \mathrm{m}$ BzATP for $1 \mathrm{~s}$ followed by $40 \mathrm{~s}$ stimulation (left). Deactivation components of each pulse normalized by the maximum current amplitude are displayed on the right. $\boldsymbol{E}$, Simulation model cell periodically stimulated with $100 \mu \mathrm{m} / 1 \mathrm{~s}$ BzATP (left). Deactivation components of each pulse normalized by the maximum current amplitude are displayed on the right. $F$, Current simulation from a model cell initially stimulated for $40 \mathrm{~s}$ with $320 \mu \mathrm{m}$ BzATP followed by $40 \mathrm{~s}$ stimulation with 100, 32 and 80 s stimulation with $10 \mu \mathrm{m}$ BzATP. As observed in the experiment, stimulation with $320 \mu \mathrm{m}$ BzATP for 40 s led to receptor sensitization and monodirectional type of current response during BZATP stimulation applied later at lower doses, a behavior not exhibited by naive receptors.

agonist concentrations. Finally, the P2X7R-specific antagonist Az10606120 rapidly abolishes $I_{2}$, further supporting the view that it is unlikely to be from some conductance other than P2X7R. These experiments do not exclude the possibility of a two-pore model, in which one pore accounts for cation permeability and can dilate and the other pore accounts for dye uptake, but suggest that a relatively rapid activation of the channel pore permeable for small inorganic cations causes $I_{1}$ growth, accompanied by a progressive pore dilation causing $I_{2}$ growth.

Experiments with repetitive stimulation with the same agonist concentration further revealed that gating of P2X7R is more complex than the presence of two conductivity states. The peak current amplitude increased and the kinetics of receptor deactivation became slower during repetitive stimulation. Once a steady current was reached, used P2X7Rs behaved like other P2XRs during subsequent stimulation, showing monophasic currents with amplitudes determined by agonist concentrations, provided the washout periods were $<30 \mathrm{~min}$. These findings are in general agreement with a recent publication describing facilitation of rat P2X7R (Roger et al., 2008). We termed this receptor sensitization, based on a finding of changes in the rates of receptor deactivation, because deactivation of other P2XRs does not change with changes in agonist concentration and repetitive stimulation (Yan et al., 2006). Thus, differences in the potency of agonist to activate P2X7R during initial and repetitive stimulation, combined with slower receptor deactivation during repetitive agonist application, suggest that P2X7R exists in two states, unsensitized and sensitized. Because the sensitized state lasts for a prolonged period, repetitive agonist applications with shorter washout period cause most of receptors to go to the sensitized state.

We do not know the mechanism of P2X7R sensitization. A role of a calciumdependent calmodulin binding motif in the $\mathrm{C}$ terminus of $\mathrm{P} 2 \mathrm{X} 7 \mathrm{R}$ in receptor gating has been proposed, causing the leftward shift in sensitivity of P2X7R for agonists (Roger et al., 2008). Our experiments do not argue against this hypothesis, but show that generation of biphasic currents and transition from biphasic to monophasic signaling is independent of bath $\mathrm{Ca}^{2+}$ and $\mathrm{Na}^{+}$concentration. The substitution of the Thr-15 residue with charged residues resulted in mutants that immediately responded with openings of the pore to a dilated state without prior ligand exposure (Yan et al., 2008). The present data show further that these mutants always responded to agonist application with amplitude-modulated monophasic current and were more sensitive to BzATP than the wild-type receptors, as indicated by decrease in the $\mathrm{EC}_{50}$ value for BzATP and a slow rate of deactivation that did not change further with repetitive agonist application. However, it is unlikely that phosphorylation of this particular residue accounts for the sensitization of receptors, because the K17A mutant behaved similarly as the wild-type receptor. What these experiments show is that the sensitized state and the dilated state reflect the same status of the receptor; sensitization describes changes in its binding/gating property and the dilation describes the accompanying changes in pore selectivity.

The crystal structure of the zebrafish P2X4R in closed state is consistent with a hypothesis that the receptor is a symmetrical trimer (Kawate et al., 2009). It has been suggested that the occupancy of all three binding sites is needed for activation of these receptors, based on studies on the initial slope of ATP concentrationresponse curves (Bean et al., 1990) and Hill plots (Jiang et al., 2003). Two kinetic models for activation of P2XRs have been proposed, which reproduced monophasic currents (Bean et al., 1990; Riedel et al., 2007). Until now no model has been put forward that can explain the complex pattern of P2X7R gating, including the increase in $I_{1}$ and decrease in $I_{2}$ amplitude with elevation in agonist concentration, or the transition from biphasic to monophasic signaling during repetitive agonist application. Here we modeled the channel as a symmetric trimer that becomes asymmetric because of negative cooperativity when one ATP binds, incorporating the essence of the two-activation site model (Klapperstück et al., 2001).

Figure 7 describes these transitions in detail. When no ATP is bound, the molecule is symmetrical and closed. When one ATP is bound, the molecule is distorted in such a way as to reduce the 
affinity of the remaining binding sites and remains closed. When a second ATP is bound, the molecule is further distorted, additionally decreasing the affinity of the third site but the channel opens in its low-conductance state. A fivefold difference in the potency of agonist for $I_{1}$ and $I_{2}$ growth supports the concept of asymmetry, and small amplitude monophasic currents observed at low agonist concentration in naive receptors support the presence of a low-conductance state. When the third ATP binds, symmetry is restored, putatively relieving the mechanical stress. We suggest that this both increases the affinity of the binding sites to ATP and causes the pore to dilate to the high conductance state. The restoration of symmetry may facilitate an unknown persistent change of state that is required for dilation.

An interesting feature of this scheme is that it factorizes the complex sequence of steps into two semi-independent processes: horizontal motion in the scheme corresponds to ATP binding and channel opening or closing, whereas vertical motion corresponds to sensitization and dilation. We assume that dilation and sensitization are two faces of the same coin and both follow the binding of the third ATP. The vertical steps must be slow to explain both the slow component of the rise of the current (i.e., $I_{2}$ current) and the memory of past exposure to high ATP, which requires tens of minutes to wash out. Whatever the biochemical nature of the sensitization/dilation step, its slowness compared with ATP binding means that the states can be written as a product of two factors, analogous to the $m$ and $h$ processes in the Hodgkin-Huxley $\mathrm{Na}^{+}$channel in the same way as previously described for the Keizer-DeYoung model (Li and Rinzel, 1994).

Other assignments of opening and conductance states to the various ATP-binding configurations are possible, and we have not shown ours are optimal. The model, however, is simple and does a good job of capturing most of the data from a wide range of experiments. It also provides a substrate for the opposite effects of P2X7R on cellular functions, such as cell growth and differentiation (occupancy of two ATP binding sites) vs cell death (occupancy of 3 ATP binding sites accompanied by pore dilation). The model itself does not confirm our main hypothesis that the kinetics of P2X7R reflects the dilation of a single channel to a persistent sensitized state. Indeed, the scheme of Figure 7 could be interpreted as the states of a two-channel complex, with the bottom row corresponding to the second pore. However, Figure 3, D-G, then requires the second pore to close rapidly when $\mathrm{P} 2 \mathrm{X} 7 \mathrm{R}$ is blocked, which seems implausible. Finally, the model can explain the complex effects of Az10606120 assuming a single type of binding site to which the compound and ATP can bind, with Az10606120 before ATP preventing ATP binding.

\section{References}

Bean BP, Williams CA, Ceelen PW (1990) ATP-activated channels in rat and bullfrog sensory neurons: current-voltage relation and singlechannel behavior. J Neurosci 10:11-19.

Becker D, Wolterdorf R, Boldt W, Schmitz S, Braam U, Schmalzing G, Markardt F (2008) The P2X7 carboxyl tail is a regulatory module of P2X7 receptor channel activity. J Biol Chem 283:25725-25734.

Chessell IP, Grahames CBA, Michel AD, Humphrey PPA (2001) Dynamics of $\mathrm{P}_{2} \mathrm{X}_{7}$ receptor pore dilation: pharmacological and functional consequences. Drug Dev Res 53:60-65.

Di Virgilio F, Ferrari D, Adinolfi E (2009a) P2X(7): a growth-promoting receptor-implications for cancer. Purinergic Signal 5:251-256.

Di Virgilio F, Ceruti S, Bramanti P, Abbracchio MP (2009b) Purinergic signalling in inflammation of the central nervous system. Trends Neurosci 32:79-87.

Elliott JI, Surprenant A, Marelli-Berg FM, Cooper JC, Cassady-Cain RL, Wooding C, Linton K, Alexander DR, Higgins CF (2005) Membrane phosphatidylserine distribution as a non-apoptotic signalling mechanism in lymphocytes. Nat Cell Biol 7:808-816.
Faria RX, Defarias FP, Alves LA (2005) Are second messengers crucial for opening the pore associated with P2X7 receptor? Am J Physiol Cell Physiol 288:C260-C271.

He ML, Zemkova H, Koshimizu TA, Tomić M, Stojilkovic SS (2003) Intracellular calcium measurements as a method in studies on activity of purinergic P2X receptor channels. Am J Physiol Cell Physiol 285:C467-C479.

Jiang LH, Kim M, Spelta V, Bo X, Surprenant A, North RA (2003) Subunit arrangement in P2X receptors. J Neurosci 23:8903-8910.

Kawate T, Michel JC, Birdsong WT, Gouaux E (2009) Crystal structure of the ATP-gated P2X(4) ion channel in the closed state. Nature 460:592-598.

Klapperstück M, Büttner C, Böhm T, Schmalzing G, Markwardt F (2000) Characteristics of P2X7 receptors from human B lymphocytes expressed in Xenopus oocytes. Biochim Biophys Acta 1467:444-456.

Klapperstück M, Büttner C, Schmalzing G, Markwardt F (2001) Functional evidence of distinct ATP activation sites at the human P2X(7) receptor. J Physiol 534:25-35.

Koshimizu TA, Van Goor F, Tomić M, Wong AO, Tanoue A, Tsujimoto G, Stojilkovic SS (2000) Characterization of calcium signaling by purinergic receptor-channels expressed in excitable cells. Mol Pharmacol 58:936-945.

Li YX, Rinzel J (1994) Equations for InsP3 receptor-mediated $\left[\mathrm{Ca}^{2+}\right]$ i oscillations derived from a detailed kinetic model: a Hodgkin-Huxley like formalism. J Theor Biol 166:461-473.

Locovei S, Scemes E, Qiu F, Spray DC, Dahl G (2007) Pannexin1 is part of the pore forming unit of the $\mathrm{P} 2 \mathrm{X}(7)$ receptor death complex. FEBS Lett 581:483-488.

Michel AD, Chambers LJ, Clay WC, Condreay JP, Walter DS, Chessell IP (2007) Direct labelling of the human P2X7 receptor and identification of positive and negative cooperativity of binding. Br J Pharmacol 151:103-114.

Monif M, Reid CA, Powell KL, Smart ML, Williams DA (2009) The P2X7 receptor drives microglial activation and proliferation: a trophic role for P2X7R pore. J Neurosci 29:3781-3791.

North RA (2002) Molecular physiology of P2X receptors. Physiol Rev 82:1013-1067.

Pelegrin P, Surprenant A (2006) Pannexin-1 mediates large pore formation and interleukin-1beta release by the ATP-gated P2X7 receptor. EMBO J 25:5071-5082.

Pelegrin P, Surprenant A (2009) The P2X(7) receptor-pannexin connection to dye uptake and IL-1beta release. Purinergic Signal 5:129-137.

Petrou S, Ugur M, Drummond RM, Singer JJ, Walsh JV Jr (1997) P2X7 purinoceptor expression in Xenopus oocytes is not sufficient to produce a pore-forming P2Z-like phenotype. FEBS Lett 411:339-345.

Rassendren F, Buell GN, Virginio C, Collo G, North RA, Surprenant A (1997) The permeabilizing ATP receptor, P2X7. Cloning and expression of a human cDNA. J Biol Chem 272:5482-5486.

Riedel T, Lozinsky I, Schmalzing G, Markwardt F (2007) Kinetics of P2X7 receptor-operated single channels currents. Biophys J 92:2377-2391.

Roger S, Pelegrin P, Surprenant A (2008) Facilitation of P2X7 receptor currents and membrane blebbing via constitutive and dynamic calmodulin binding. J Neurosci 28:6393-6401.

Skaper SD, Debetto P, Giusti P (2010) The P2X7 purinergic receptor: from physiology to neurological disorders. FASEB J 24:337-345.

Smart ML, Gu B, Panchal RG, Wiley J, Cromer B, Williams DA, Petrou S (2003) P2X7 receptor cell surface expression and cytolytic pore formation are regulated by a distal C-terminal region. J Biol Chem 278:8853-8860.

Surprenant A, North RA (2009) Signaling at Purinergic P2X Receptors. Annu Rev Physiol 71:333-359.

Surprenant A, Rassendren F, Kawashima E, North RA, Buell G (1996) The cytolytic P2Z receptor for extracellular ATP identified as a P2X receptor (P2X7). Science 272:735-738.

Virginio C, MacKenzie A, North RA, Surprenant A (1999) Kinetics of cell lysis, dye uptake and permeability changes in cells expressing the rat P2X7 receptor. J Physiol 519:335-346.

Yan Z, Liang Z, Tomic M, Obsil T, Stojilkovic SS (2005) Molecular determinants of the agonist binding domain of a P2X receptor channel. Mol Pharmacol 67:1078-1088.

Yan Z, Liang Z, Obsil T, Stojilkovic SS (2006) Participation of the Lys313-Ile333 sequence of the purinergic $\mathrm{P} 2 \mathrm{X} 4$ receptor in agonist binding and transduction of signals to the channel gate. J Biol Chem 281:32649-32659.

Yan Z, Li S, Liang Z, Tomić M, Stojilkovic SS (2008) The P2X7 receptor channel pore dilates under physiological ion conditions. J Gen Physiol 132:563-573. 